\title{
A PERTURBATION METHOD IN CRITICAL POINT THEORY AND APPLICATIONS \\ BY
}

\author{
ABBAS BAHRI AND HENRI BERESTYCKI
}

\begin{abstract}
This paper is concerned with existence and multiplicity results for nonlinear elliptic equations of the type $-\Delta u=|u|^{p-1} u+h(x)$ in $\Omega, u=0$ on $\partial \Omega$. Here, $\Omega \subset \mathbf{R}^{N}$ is smooth and bounded, and $h \in L^{2}(\Omega)$ is given. We show that there exists $p_{N}>1$ such that for any $p \in\left(1, p_{N}\right)$ and any $h \in L^{2}(\Omega)$, the preceding equation possesses infinitely many distinct solutions.

The method rests on a characterization of the existence of critical values by means of noncontractibility properties of certain level sets. A perturbation argument enables one to use the properties of some associated even functional. Several other applications of this method are also presented.
\end{abstract}

1. Introduction. In this paper, we study nonlinear elliptic problems of the type

$$
\left\{\begin{array}{l}
-\Delta u=|u|^{p-1} u+h(x) \text { in } \Omega, \\
u=0 \text { on } \partial \Omega .
\end{array}\right.
$$

Here, $\Omega \subset \mathbf{R}^{N}$ is a smooth and bounded domain, $p>1$, and $h$ is some given function in $L^{2}(\Omega)$.

In the case (1.1) is odd, that is $h \equiv 0$, and $1<p<(N+2) /(N-2)$ if $N \geqslant 3$ or $1<p<+\infty$ if $N=2$, it is well known that (1.1) has infinitely many distinct solutions $\left(u_{k}\right)_{k \in N}$ associated with critical values $I^{*}\left(u_{k}\right)$ of the functional

$$
I^{*}(u)=\frac{1}{2} \int_{\Omega}|\nabla u|^{2} d x-\frac{1}{p+1} \int_{\Omega}|u|^{p+1} d x
$$

such that $\lim _{k \rightarrow+\infty} I^{*}\left(u_{k}\right)=+\infty$. Results of this kind were obtained by Coffman [10], Hempel [16], Ambrosetti [3], Rabinowitz [21], and for the most general odd nonlinearities by Ambrosetti and Rabinowitz [4] and Rabinowitz [22]. In these works, the methods rely on the use of Lusternik-Schnirelman theory or rather on the notion of genus for symmetric sets. Therefore, the fact that (1.1) is odd (or equivalently that $I^{*}$ is even) is essential for applying these techniques.

A natural and open question is to know whether the infinite number of solutions persists under perturbations of the odd equation. In particular, does (1.1) possess infinitely many solutions when $h \neq 0$ for values of $p$ in the same range as before? Actually, even the existence of at least one solution of (1.1) is not known when $N \geqslant 2$, for arbitrary $h \in L^{2}(\Omega)$. The only previous result in that direction for

Received by the editors July 25, 1980.

1980 Mathematics Subject Classification. Primary 35J60; Secondary 58E05.

Key words and phrases. Nonlinear elliptic partial differential equation, critical point, perturbation, contractible set, level set, even and noneven functional, multiplicity.

(C) 1981 American Mathematical Society 0002-9947/81/0000-0400/\$09.00 
$N \geqslant 2$ is, to our knowledge, a perturbation theorem of A. Ambrosetti [2] stating, in the case of (1.1), that for any number $\nu \in N$, there exists $\varepsilon_{\nu}>0$ such that if $\|h\|_{L^{2}(\Omega)} \leqslant \varepsilon_{\nu}$, then problem (1.1) has at least $\nu$ distinct solutions. A much more general perturbation-multiplicity result will be derived here in $§ 7$. Let us remark that the existence of at least one solution of (1.1) for $\|h\|_{L^{2}(\Omega)}$ small enough is a straightforward application of the implicit function theorem.

The results we obtain for (1.1) can be considered as a first and partial answer to the questions mentioned above. We denote by $I$ the energy associated with (1.1):

$$
I(u)=\frac{1}{2} \int_{\Omega}|\nabla u|^{2} d x-\frac{1}{p+1} \int_{\Omega}|u|^{p+1} d x-\int_{\Omega} h u d x .
$$

Our main result for $(1.1)$ is

THEOREM 1.1. For all $N \geqslant 2$, denote by $p_{N}$ the largest root of the equation $(2 N-2) p^{2}-(N+2) p-N=0,\left(p_{N}>1\right)$. For any $p \in\left(1, p_{N}\right)$ and for any $h \in$ $L^{2}(\Omega)$ problem (1.1) has infinitely many distinct solutions $\left(u_{k}\right), k \in \mathbf{N}$, such that $\lim _{k \rightarrow+\infty} I\left(u_{k}\right)=+\infty$.

REMARK 1.2. In dimension $N=1$, a much more general result was established by Ehrmann [14] and Fučik and Lovicar [15]. They show that the ordinary differential equation (in $y=y(x)$ )

$$
\left\{\begin{array}{l}
-y^{\prime \prime}=f(y)+h(x), \quad x \in(0,1), \\
y(0)=y(1)=0
\end{array}\right.
$$

has infinitely many distinct solutions under essentially the sole assumption

$$
\lim _{y \rightarrow \pm \infty} \frac{f(y)}{y}=+\infty .
$$

The method employed in [14], [15] is purely O.D.E. in nature: It relies on a "shooting" argument combined with counting the oscillations of the solutions of (1.4) on $(0,1)$. Thus, it cannot be extended neither to higher dimensions, nor, at least seemingly, to systems of ordinary differential equations, nor to nonlocal equations in one variable.

REMARK 1.3. It is easily checked that one always has

$$
1<p_{N}<(N+2) /(N-2) \text {. }
$$

Complementary results to Theorem 1.1 for (1.1) have been obtained by one of the authors (A. Bahri [5], [6]). He shows in particular that for any $p$, such that $1<p<(N+2) /(N-2)$ if $N \geqslant 3$ (and $1<p$ if $N=2$ ), problem (1.1) has infinitely many solutions for a dense set of $h$ in $H^{-1}(\Omega)$. Actually, a stronger result is derived in [5], [6]. Defining an $\varepsilon$-solution of $(1.1)(\varepsilon>0)$ as a function $u \in H_{0}^{1}(\Omega)$ such that

$$
\left\|\Delta u+|u|^{p-1} u+h(x)\right\|_{H^{-1}(\Omega)}<\varepsilon,
$$

it is proved in [5], [6] that for any $\varepsilon>0$, (1.1) possesses infinitely many distinct $\varepsilon$-solutions. (We also refer to [5], [6] for a new proof of the fact that (1.1) has infinitely many distinct solutions in the odd case, i.e. $h \equiv 0$, which uses an application of Brouwer's fixed point theorem.) 
The preceding result leads us to believe that the definition of $p_{N}$ in Theorem 1.1 is only due to a limitation of the method we use. It seems natural to conjecture that the result of Theorem 1.1 remains valid for any $p \in(1,(N+2) /(N-2))$ when $N \geqslant 3$ and any $p>1$ when $N=2$.

REMARK 1.4. Existence of critical values for perturbations of even functionals has been studied in Krasnosel'skii [17], Ambrosetti [2] and Marino and Prodi [18]. (In the latter, one can also find applications to some fourth order elliptic equations.)

The proof of Theorem 1.1 will be delayed until $\S 5$. Rather than working directly with the functional $I(u)$ defined in (1.3), we will prove the existence of critical values for the "constrained" functional

$$
J(u)=\max _{\lambda>0} I(\lambda u)
$$

defined on the sphere $S=\left\{u \in H_{0}^{1}(\Omega), \int_{\Omega}|\nabla u|^{2} d x=1\right\}$. Indeed, we show in $\$ 4$ that the positive critical values of $J$ (on $S$ ) and $I$ (in $H_{0}^{1}(\Omega)$ ) are the same. In the next two sections we derive some general properties concerning the critical values of a functional on a sphere. The principle for perturbing critical values of an even functional is stated in $\$ 3$.

We prove the same result as Theorem 1.1 for (1.1) with an odd nonlinearity $g(x, u)$ more general than $|u|^{p-1} u$ in $\$ 6$. We have nevertheless separated the particular case $g(u)=|u|^{p-1} u$ because the arguments are somewhat more transparent in this case. Another application of this method to a "perturbation and multiplicity" result for nonlinear elliptic equations is presented in §7. In forthcoming papers [8], [9], we apply some related methods to prove the existence of infinitely many distinct periodic solutions of some Hamiltonian systems in the presence of periodic "forcing" terms.

The paper is organized as follows:

1. Introduction.

2. Contractibility properties and critical values.

3. Perturbation of critical values of an even functional.

4. A functional on the sphere associated with (1.1).

5. Proof of Theorem 1.1.

6. More general nonlinearities.

7. A perturbation and multiplicity result.

Some of the results presented here have been announced in [7].

The authors are much thankful to Professor P. H. Rabinowitz for several stimulating discussions on this work and on many related subjects. ${ }^{1}$

2. Contractibility properties and critical values. Let $H$ be a real Hilbert space with norm $\|\cdot\|$. Let $S=\{x \in H ;\|x\|=1\}$ denote the unit sphere. We consider a

\footnotetext{
${ }^{1}$ After this manuscript was completed the authors have learned from Professor P. H. Rabinowitz that results which are related to Theorem 1.1 and to those of $\$ 6$ have been obtained independently by Michael Struwe.
} 
functional $J \in C^{2}(S, \mathbf{R}) . J$ will be assumed to satisfy the following Palais-Smale condition:

$\left\{\begin{array}{l}\text { For any sequence }\left(x_{n}\right) \subset S \text { such that } J\left(x_{n}\right) \text { is bounded and } \\ \left\|J^{\prime}\left(x_{n}\right)\right\| \rightarrow 0 \text {, one can extract from }\left(x_{n}\right) \text { a convergent subsequence. }\end{array}\right.$

In this condition, the norm $\left\|J^{\prime}\left(x_{n}\right)\right\|$ is taken in the cotangent space $\left(T_{x_{n}} S\right)^{\prime}$ as the dual norm, the tangent space $T_{x_{n}} S$ being always equipped with the norm inherited from $H$. Most of the time, $J$ will only be required to satisfy the following weaker condition than (P.S):

$\left\{\begin{array}{l}\text { For any } C>a \text { and for any sequence, }\left(x_{n}\right) \subset S \text { such that } a \leqslant J\left(x_{n}\right) \leqslant C \\ \text { and }\end{array}\right.$

where $a \in \mathbf{R}$ is some number.

In the following, for $a \in \mathbf{R}$, we denote

$$
J_{a}=\{x \in S ; J(x) \leqslant a\}, \quad \tilde{J}_{a}=\{x \in S ; J(x) \geqslant a\} .
$$

The following result is a basic tool in the sequel for finding critical values.

Theorem 2.1. Suppose $H$ is infinite dimensional and $J \in C^{2}(S, \mathbf{R})$ satisfies condition (P.S). If $J_{a}$ is not contractible in itself to a point, then $J$ has a critical value in $[a,+\infty)$.

The proof of Theorem 2.1 is by contradiction. We first require the following classical lemma.

LemMA 2.2. Let $J \in C^{2}(S, \mathbf{R})$ satisfy condition (P.S) and let $a \in \mathbf{R}$ be such that $J$ has no critical values in $[a,+\infty)$. Then, $J_{a}$ is a (deformation) retract of $S$.

Proof of Lemma 2.2. (See Palais [19], [20], Marino and Prodi [18] and Rabinowitz [22] for similar results and detailed proofs.) We just sketch the argument here. Let $\varepsilon>0$ be such that $J$ has no critical values in $[a-\varepsilon,+\infty$ ) (the existence of such an $\varepsilon$ is a direct consequence of (P.S)). Let $\chi$ be a locally Lipschitz function such that $0 \leqslant \chi \leqslant 1, \chi \equiv 0$ on $J_{a-\varepsilon}$ and $\chi \equiv 1$ on $\tilde{J}_{a}$. Let $\eta(t, x)$ denote the solution of the following differential equation on $S$ :

$$
d \eta / d t=-\chi(\eta) J^{\prime}(\eta) /\left\|J^{\prime}(\eta)\right\|^{2} ; \quad \eta(0, x)=x .
$$

It is easily checked that due to the fact that $J$ is of class $C^{2}$, satisfies (P.S) and has no critical values in $[a-\varepsilon,+\infty), \eta(t, x)$ is defined for all $t \geqslant 0$. Furthermore, $x \rightarrow \eta(t, x)$ is a homeomorphism of $S$ for any $t \geqslant 0, \eta(t, x)=x, \forall x \in J_{a-e}$, $t \geqslant 0, J(\eta(t, x))$ is nonincreasing with respect to $t$ and

$$
d J(\eta(t, x)) / d t=-1 \quad \text { if } \eta(t, x) \in \tilde{J}_{a} .
$$

Set

$$
r(x)=\eta\left((J(x)-a)^{+}, x\right) \text { for } x \in S,
$$

where $m^{+}=\max (m, 0)$. Then, $r \in C\left(S, J_{a}\right)$ and $r_{\mid J_{a}}=\operatorname{Id}_{\left.\right|_{a}}$ (the identity on $J_{a}$ ). Indeed, if $x \in J_{a}$, then $r(x)=\eta(0, x)=x$, while if $x \in \tilde{J}_{a}$, then it is easily verified (using (2.2)) that $J(r(x))-J(x)=-(J(x)-a)$, that is $J(r(x))=a$. 
Observe that $J_{a}$ actually is a strong deformation retract of $S$. It just suffices to set

$$
r_{\tau}(x)=\eta\left(\tau(J(x)-a)^{+}, x\right), \quad \tau \in[0,1], x \in S,
$$

to have the desired retraction by deformation that leaves $J_{a}$ invariant.

Proof of Theorem 2.1. By Dugundji's theorem [13], if $H$ is infinite dimensional, then $S$ is contractible in itself to a point. Thus, if $J$ has no critical values in $[a,+\infty)$, then $J_{a}$ being a retract of $S$ is also contractible in itself to a point. Indeed, let $\phi \in C([0,1] \times S, S)$ be such that $\phi(0, x)=x$ and $\phi(1, x)=x_{0}\left(x_{0} \in S\right.$ is fixed). Set $\psi(t, x)=r[\phi(t, x)]$, where $r$ is given by Lemma 2.2. Then,

$$
\psi \in C\left([0,1] \times J_{a}, J_{a}\right)
$$

and satisfies $\psi(0, x)=x$ for $x \in J_{a}$ and $\psi(1, x)=r\left(x_{0}\right), \forall x \in J_{a}$.

Therefore, if $J$ has no critical values in $[a,+\infty)$, the set $J_{a}$ is contractible in itself to a point. The proof of Theorem 2.1 is thereby complete.

RemarK 2.3. Suppose $a \in \mathbf{R}$ is not a critical value of $J$ (satisfying the hypotheses of Theorem 2.1) and $J_{a}$ is not contractible in itself to a point. Suppose furthermore that there exists $A>a$ such that $J_{a}$ is contractible to a point in $J_{A}$. Then, there is a "variational characterization" of a critical value of $J$ above $a$. Indeed, setting

$$
c=\inf \left\{b>a ; J_{a} \text { is contractible to a point in } J_{b}\right\}
$$

or

$$
\hat{c}=\sup \left\{b>a ; J_{a} \text { is not contractible to a point in } J_{b}\right\}
$$

it is easily seen that $a<c=\hat{c} \leqslant A$ and that $c=\hat{c}$ is a critical value of $J$. (Notice that since $a$ is not a critical value and $J_{a}$ is not contractible in itself to a point, then $J_{a}$ is not contractible in $J_{b}$ for $b>a$ and $b$ sufficiently near to $a$.)

REMARK 2.4. It is readily checked by inspection of the proof of Lemma 2.2 that the conclusion of Theorem 2.1 remains valid if one only assumes that $J \in$ $C^{0}(S, \mathbf{R}), J \in C^{2}\left(\tilde{J}_{a^{\prime}}, \mathbf{R}\right)$ and $J$ verifies condition (P.S) $)_{a^{\prime}}$ for some $a^{\prime}<a$.

From the viewpoint of the applications to nonlinear partial differential equations, it is desirable to weaken the assumption that $J$ be of class $C^{2}$. We then obtain the following slightly weaker result for $C^{1}$ functionals.

Theorem 2.5. Suppose $H$ is infinite dimensional, $J \in C^{0}(S, \mathbf{R}), J \in C^{1}\left(\tilde{J}_{a}, \mathbf{R}\right)$, and $J$ satisfies condition (P.S $)_{a}$. If there exists some $\varepsilon>0$ such that $J_{a}$ is not contractible in $J_{a+\varepsilon}$ to a point, then $J$ has a critical value in $[a,+\infty)$.

To prove Theorem 2.5 we will show that if $J \in C^{1}\left(\tilde{J}_{a}, \mathbf{R}\right)$ satisfies (P.S $)_{a}$ and $J$ has no critical value in $[a,+\infty)$, then for any $\varepsilon>0, J_{a}$ is contractible to a point in $J_{a+\varepsilon}$. This will be derived from the following modification of Lemma 2.2.

Lemma 2.6. Let $J \in C^{0}(S, \mathbf{R}), J \in C^{1}\left(\tilde{J}_{a}, \mathbf{R}\right)$ and $J$ satisfies (P.S $)_{a}$. Suppose $J$ has no critical values in $[a,+\infty)$. Then, for any $\varepsilon>0$, there exists $r_{\varepsilon} \in C\left(S, J_{a+\varepsilon}\right)$ such that $r(x)=x$ for all $x \in J_{a}$.

Proof of Lemma 2.6. (See, e.g., Palais [19], [20], Rabinowitz [22] for similar results.) Since $J$ is no longer $C^{2}$, we cannot directly define the flow $\eta(t, x)$ by $(2.1)$. We use instead of $J^{\prime}$, the pseudo-gradient vector field on $\tilde{J}_{a+e_{1}}$ where $\varepsilon_{1}$ is a fixed 
real number such that $0<\varepsilon_{1}<\varepsilon$. We recall (e.g. from Palais [19]) that there exists a locally Lipschitz vector field on $\tilde{J}_{a+\varepsilon_{1}}, v(x) \in T_{x} S$ such that

$$
\left\{\begin{array}{l}
\|v(x)\| \leqslant 2\left\|J^{\prime}(x)\right\| \\
\left\langle J^{\prime}(x), v(x)\right\rangle \geqslant\left\|J^{\prime}(x)\right\|^{2}
\end{array} \quad \forall x \in \tilde{J}_{a+\varepsilon_{1}} .\right.
$$

Since $J$ has no critical values in $[a,+\infty)$, we have from $(2.3),\|v(x)\| \geqslant\left\|J^{\prime}(x)\right\|$ $>0, \forall x \in \tilde{J}_{a+\varepsilon_{1}}$. Let $\chi: S \rightarrow \mathbf{R}$ be a locally Lipschitz function such that $0 \leqslant \chi \leqslant$ $1, \chi \equiv 0$ on $J_{a+\varepsilon_{1}}$ and $\chi \equiv 1$ on $\tilde{J}_{a+\varepsilon}$. Set

$$
\left\{\begin{array}{l}
V(\eta)=-\chi(\eta) v(\eta) /\|v(\eta)\|^{2} \quad \text { for } \eta \in \tilde{J}_{a+\varepsilon_{1}} \\
V(\eta)=0 \text { for } \eta \in J_{a+\varepsilon_{1}}
\end{array}\right.
$$

$V$ is a locally Lipschitz vector field on $S$. We consider the flow $\eta(t, x)$ generated by $V$, that is, $\eta(t, x)$ is the solution of the following equation on $S$

$$
d \eta / d t=V(\eta), \quad \eta(0, x)=x .
$$

From (2.3) and (2.4) it follows that

$$
\frac{d J(\eta)}{d t}=-\chi(\eta) \frac{\left\langle J^{\prime}(\eta), v(\eta)\right\rangle}{\|v(\eta)\|^{2}} \leqslant-\chi(\eta) \leqslant 0 .
$$

Since $V$ is locally Lipschitz, $\eta(t, x)$ is uniquely defined in (2.5) on some maximal time interval $0 \leqslant t<\bar{t}(x)$, and is continuous with respect to $t$ and $x$. Now, for $x \in J_{a+\varepsilon_{1}}$, one has $\eta(t, x)=x, \forall t \geqslant 0$ and $\bar{t}(x)=+\infty$. For $x \in \tilde{J}_{a+\varepsilon_{1}}$, one derives from (2.6) that $a+\varepsilon_{1} \leqslant J(\eta(t, x)) \leqslant J(x), \forall t \in[0, \bar{t}(x))$. (The left-hand side inequality is derived from $V \equiv 0$ on $J_{a+\varepsilon_{1}}$.) Since $J$ has no critical values in $[a,+\infty)$, it is easily derived from condition (P.S) ${ }_{a}$ (see, e.g., Rabinowitz [22]) that there exists $\delta=\delta(x)>0$ such that $\forall y \in S$ with $a+\varepsilon_{1} \leqslant J(y) \leqslant J(x)$, one has $\left\|J^{\prime}(y)\right\| \geqslant \delta(x)$. Hence, for any $x \in \tilde{J}_{a+\varepsilon_{1}}$,

$$
\|v(\eta(t, x))\| \geqslant\left\|J^{\prime}(\eta(t, x))\right\| \geqslant \delta(x)>0, \quad \forall t \geqslant 0 .
$$

Thus,

$$
\|V(\eta(t, x))\| \leqslant 1 /\|v(\eta(t, x))\| \leqslant 1 / \delta(x), \quad \forall t \geqslant 0 .
$$

This implies that in (2.5), $\eta(t, x)$ is defined for all $t \geqslant 0$ : Thus, $\bar{t}(x)=+\infty$, $\forall x \in S$.

Therefore, $x \rightarrow \eta(t, x)$ is continuous: $S \rightarrow S$ for any $t \geqslant 0$, and is such that $\eta(t, x)=x, \forall t \geqslant 0, \forall x \in J_{a+\varepsilon_{1}} \cdot J(\eta(t, x))$ is nonincreasing with respect to $t$. Now set

$$
r_{\varepsilon}(x)=\eta\left((J(x)-a)^{+}, x\right)
$$

$r_{\varepsilon} \in C(S, S), r_{\varepsilon}(x)=x, \forall x \in J_{a}$ and

$$
J\left(r_{\varepsilon}(x)\right) \leqslant J(\eta(t, x)) \leqslant J(x), \quad \forall t, 0 \leqslant t \leqslant(J(x)-a)^{+} .
$$

Let us show that $r_{\varepsilon}(S) \subset J_{a+\varepsilon}$. From (2.8) it is clear that $r_{\varepsilon}\left(J_{a+\varepsilon}\right) \subset J_{a+\varepsilon}$. So suppose $x \in \tilde{J}_{a+\varepsilon}$. We claim $r_{\varepsilon}(x) \in J_{a+\varepsilon}$. For if not, we have by $(2.8), \eta(t, x) \in$ $\tilde{J}_{a+\varepsilon}$ for all $t, 0 \leqslant t \leqslant(J(x)-a)$. Whence, $\chi(\eta(t, x))=1, \forall t, 0 \leqslant t \leqslant(J(x)-a)$. From (2.6) we then derive

$$
J\left(r_{\varepsilon}(x)\right)-J(x) \leqslant-(J(x)-a)
$$


that is, $J\left(r_{\varepsilon}(x)\right) \leqslant a$ which is a contradiction. Thus, $r_{\varepsilon}(S) \subset J_{a+\varepsilon}$, and the proof of Lemma 2.6 is complete.

REMARK 2.7. Clearly, $r_{\varepsilon}$ is homotopic to the identity on $S$ by means of a homotopy $\left(r_{\varepsilon}\right)_{\tau}, \tau \in[0,1]$, such that $\left(r_{\varepsilon}\right)_{\tau}$ is the identity on $J_{a}, \forall \tau \in[0,1]$.

Proof of TheOREM 2.5. Let $\phi$ be a contraction of $S$ in itself to a point as in the proof of Theorem 2.1. Let us define $\psi_{\varepsilon} \in C\left([0,1] \times J_{a}, J_{a+\varepsilon}\right)$ by setting $\psi_{\varepsilon}(t, x)=$ $r_{\varepsilon}(\phi(t, x))$.

Clearly $\psi_{\varepsilon}\left([0,1] \times J_{a}\right) \subset J_{a+\varepsilon}\left(\right.$ since $\left.r_{\varepsilon}(S) \subset J_{a+\varepsilon}\right), \psi_{\varepsilon}(0, x)=x, \forall x \in J_{a}$ while $\psi_{e}(1, x)=r\left(x_{0}\right)$ is independent of $x$. The proof of Theorem 2.5 is thereby complete.

The preceding results just assert the existence of a critical value above a given number $a$. In applications, however, one often wishes to have some control or bound on the critical value. For instance, such a control is crucial in deriving the perturbation and multiplicity result of $\S 7$. The next two theorems provide some additional information in this direction.

Theorem 2.8. Suppose $J \in C^{0}(S, \mathbf{R}), J \in C^{1}\left(\tilde{J}_{a}, \mathbf{R}\right)$ and $J$ satisfies (P.S $)_{a}$. Furthermore, assume that there exist $\mu(a)$ and $\varepsilon>0$ with $\mu(a)>a+\varepsilon>a$ such that $J_{a}$ is contractible in $J_{\mu(a)}$ to a point and $J_{a}$ is not contractible to a point in $J_{a+\varepsilon}$. Then $J$ has a critical value in $[a, \mu(a)]$.

Proof. This result readily follows from Remark 2.3. One can also modify the argument of the preceding proof in the following manner. If $J$ has no critical value in $[a, \mu(a)]$, then (compare with Lemma 2.6) there exists $r \in C\left(J_{\mu(a)}, J_{a+\varepsilon}\right)$ with $r(x)=x, \forall x \in J_{a}$. Now, let $\phi(t, x)$ be a deformation of $J_{a}$ to a point in $J_{\mu(a)}$. Then, $\psi(t, x)=r[\phi(t, x)]$ is a deformation of $J_{a}$ to a point in $J_{a+\varepsilon}$, which is impossible.

We now derive quite a general condition which guarantees the existence of $\mu(a)$ as in Theorem 2.8, for all $a$. This result is due to A. Bahri [6]. We present here a somewhat different proof than in [6].

TheOREM 2.9. Let $\boldsymbol{H}$ be an infinite-dimensional separable Hilbert space. Suppose J is any functional on $S$ with the following property: For any sequence $\left(x_{n}\right) \subset S$, $J\left(x_{n}\right) \rightarrow+\infty$ if and only if $x_{n} \rightarrow 0$ weakly in $H$. Then, for any $a \in \mathbf{R}$, there exists $\mu(a) \geqslant a$ such that $J_{a}$ is contractible to a point in $J_{\mu(a)}$.

Proof. We first claim that there is some $e \in S$ with the property that $-\tau e$ is not in the weak closure of $J_{a}$ for any $\tau \in[0,1]$. Indeed, arguing by contradiction, let $\left(e_{n}\right) \subset S$ be a sequence such that $e_{n} \rightarrow 0$ weakly in $H$. Then, $\exists \tau_{n} \in[0,1],-\tau_{n} e_{n}$ is in the weak closure of $J_{a}$. That is, there is a sequence $\left(x_{n}\right)_{m} \subset J_{a}$ with $\left(x_{n}\right)_{m} \rightarrow$ $-\tau_{n} e_{n}$ weakly in $H$, as $m \rightarrow+\infty$. Then by a diagonal selection procedure, we can extract from $\left(x_{n}\right)_{m}$ a sequence $y_{n}=\left(x_{n}\right)_{m(n)}$ which converges weakly to 0 in $\boldsymbol{H}$. Indeed, if $\left\{\phi_{1}, \ldots, \phi_{j}, \ldots\right\}$ is a Hilbert basis of $H$, then one selects $m(n)$ in such a way that

$$
\left|\left(y_{n}+\tau_{n} e_{n}, \phi_{j}\right)\right|<1 / n \quad \forall j=1, \ldots, n .
$$


Thus $\left(y_{n}, \phi_{j}\right) \rightarrow 0$ as $n \rightarrow+\infty$ for all $j \in \mathbf{N}$, whence $y_{n} \rightarrow 0$ weakly in $H$. But since $J\left(y_{n}\right) \leqslant a$, we have reached a contradiction with the hypothesis of the theorem.

Now, let $e \in S$ be as above. We define

$$
w(t, x)=\frac{t x+(1-t) e}{\|t x+(1-t) e\|}, \quad x \in J_{a}, t \in[0,1] .
$$

Since $t x+(1-t) e \neq 0, w(t, x)$ is a continuous deformation of $J_{a}$ into a point on $S$. We claim that there exists $\mu(a) \geqslant a$ so that

$$
J(w(t, x)) \leqslant \mu(a), \quad \forall t \in[0,1], \forall x \in J_{a} .
$$

For if not, there exists $\left(x_{n}\right) \subset J_{a}, t_{n} \in[0,1]$, with $J\left(w\left(t_{n}, x_{n}\right)\right) \rightarrow+\infty$. We can assume that $t_{n} \rightarrow t \in[0,1]$ and $x_{n} \rightarrow x$ weakly in $H$. Thus, $w\left(t_{n}, x_{n}\right) \rightarrow 0$ weakly in $H$ which implies $t_{n} x_{n}+\left(1-t_{n}\right) e \rightarrow 0$ weakly in $H$, whence $t x+(1-t) e=0$. This would imply that $x=-\tau e$ with $\tau \geqslant 0$. But since $\|e\|=1$ and $\|x\| \leqslant 1$, we have $\tau \in[0,1]$. Hence, $-\tau e$ would be in the weak closure of $J_{a}$ with $\tau \in[0,1]$, which is impossible.

Therefore, $w(t, x)$ is a continuous deformation of $J_{a}$ in $J_{\mu(a)}$ to a point.

REMARK 2.10. Suppose one has a family of functionals $J^{e}$ on $S, \varepsilon \in[0,1]$, with the property that for any sequence $\left(x_{n}\right) \subset S$ and for any sequence $\left(\varepsilon_{n}\right) \subset[0,1]$, one has $J^{\varepsilon_{n}}\left(x_{n}\right) \rightarrow+\infty$ if and only if $x_{n} \rightarrow 0$ weakly in $H$. Then, as is clearly seen from the proof above, $\mu(a)$ can be chosen independently of $\varepsilon$. That is, for any $a \in \mathbf{R}$, there exists $\mu(a) \geqslant a$ such that $J_{a}^{\varepsilon}$ is contractible to a point in $J_{\mu(a)}^{\varepsilon}$ for any $\varepsilon \in[0,1]$. This observation will be useful in $\S 7$.

3. Perturbation of critical values of an even functional. In order to apply Theorems 2.1 and 2.5 for finding critical points one has to determine criteria for noncontractibility properties of the level sets of a functional on $S$. For even functionals, such a criterion is provided by Krasnosel'skii's theory of stable critical points for an even functional [17, Chapter VI]. We first recall some basic facts concerning critical points of an even functional on a sphere. We then will show in the light of the preceding section a "stability" result for these critical points allowing one to study perturbations of an even functional.

We follow here the approach of Krasnosel'skii [17, Chapter VI]. Consider the following class of compact symmetric subsets of $S$.

$$
M_{k}=\left\{A \subset S ; A=g\left(S^{k}\right) \text { where } g \text { is odd and continuous }\right\}
$$

where $k \in \mathbf{N}$ and $S^{k}=\left\{x \in \mathbf{R}^{k+1},|x|=1\right\}$ is the $k$-dimensional sphere. Let $J^{*} \in C^{1}(S, \mathbf{R})$ be an even functional. (The superscript * will always be associated with evenness thereafter.) Define

$$
C_{k}=\inf _{A \in M_{k}} \max _{x \in A} J^{*}(x) .
$$

We have the following result adapted from Krasnosel'skii [17, Chapter VI].

THEOREM 3.1. Let $J^{*} \in C^{1}(S, \mathbf{R})$ be an even functional satisfying condition (P.S) and such that $J^{*}$ is bounded from below on $S$. Let $C_{k}$ be defined by (3.2). Then:

(i) $C_{k}$ is a critical value of $J^{*}, \forall k \geqslant 0$ (if $J^{*}$ only satisfies (P.S) $)_{a}$, then $C_{k}$ is a critical value of $J^{*}$ provided that a $<C_{k}$ ). 
(ii) $-\infty<C_{k} \leqslant C_{k+1}, \forall k \geqslant 0$.

(iii) For all $a, b \in \mathbf{R}$ such that $C_{k}<b<a<C_{k+1}$, $J_{b}^{*}$ is not contractible to $a$ point in $J_{a}^{*}$.

Proof of Theorem 3.1. We omit the proofs of (i) and (ii) since they are classical (the reader is referred to Rabinowitz [22] for closely related results). We only recall here the proof of (iii). This is the crucial property that will allow us to apply the results of $\$ 2$. It is a consequence of the following lemma of Krasnosel'skii [17].

Lemma 3.2. Let $A \in M_{k}$ and suppose $\psi \in C([0,1] \times A, S)$ is a contraction of $A$ in $S$ to a point, that is $\psi(0, x)=x, \forall x \in A, \psi(1, x)=x_{0} \in S, \forall x \in A$. Set $[\psi, A]=$ $\{\psi(t, x) ; t \in[0,1], x \in A\} \cup\{-\psi(t, x) ; t \in[0,1], x \in A\}$. Then $[\psi, A] \in M_{k+1}$.

We recall the proof of Lemma 3.2. Let $A=g\left(S^{k}\right)$ with $g$ continuous and odd. We write $x \in S^{k+1}$ in the form $x=\left(x^{\prime}, t\right)$ with $x^{\prime} \in \mathbf{R}^{k+1}, t \in \mathbf{R},\left|x^{\prime}\right|^{2}+t^{2}=1$. Define a mapping $h: S^{k+1} \rightarrow[\psi, A]$ by setting

$$
h\left(x^{\prime}, t\right)= \begin{cases}x_{0}, & \text { for } t=1, x^{\prime}=0 \\ \psi\left(t, g\left(x^{\prime} /\left|x^{\prime}\right|\right)\right) & \text { for } 0 \leqslant t<1, \\ -\psi\left(|t|, g\left(-x^{\prime} /\left|x^{\prime}\right|\right)\right) & \text { for }-1<t \leqslant 0, \\ -x_{0} & \text { for } t=-1, x^{\prime}=0\end{cases}
$$

Clearly, $h\left(S^{k+1}\right)=[\psi, A], h$ is odd and continuous. Therefore $[\psi, A] \in M_{k+1}$.

Proof of (iii) Completed. Suppose $C_{k}<b<a<C_{k+1}$ and suppose by way of contradiction that $J_{b}^{*}$ is contractible to a point in $J_{a}^{*}$. Let $A \in M_{k}$ be such that $C_{k} \leqslant \max _{A} J^{*}<b$, that is, $A \subset J_{b}^{*}$. Then $A$ is contractible to a point in $J_{a}^{*}$. Let $\psi \in C\left([0,1] \times A, J_{a}^{*}\right)$ be this contraction. Since $J^{*}$ is even, one sees that $[\psi, A]$, defined in Lemma 3 , is such that $[\psi, A] \subset J_{a}^{*}$. Hence, $B=[\psi, A]$ verifies $B \in$ $M_{k+1}$ (Lemma 3.2) and $B \subset J_{a}^{*}$ or $\max _{B} J^{*}<a<C_{k+1}$, which is impossible by the very definition of $C_{k+1}$. Thus, $J_{b}^{*}$ is not contractible to a point in $J_{a}^{*}$ and the proof of (iii) is complete.

Under an additional assumption, we now show that $C_{k} \nearrow+\infty$ as $k \nearrow+\infty$.

THEOREM 3.3. In addition to the hypotheses of Theorem 3.1 suppose that $H$ is separable and that for any sequence $\left(x_{n}\right) \subset S$ such that $x_{0} \rightarrow 0$ (weakly in $H$ ) one has $J^{*}\left(x_{n}\right) \rightarrow+\infty$. Then, $\lim _{k \rightarrow+\infty} C_{k}=+\infty$.

Remark 3.4. Assume that $F^{*}(x)=1 / J^{*}(x)$ is defined on $B_{1}=\{x \in H,\|x\|<$ 1) and is such that $F^{*}(0)=0, F^{*}(x)>0$ if $x \in B_{1}-\{0\}$. Suppose further that $F^{*}$ is weakly continuous. Then $J^{*}$ satisfies the condition in Theorem 3.3. Thus, in this case it is seen at once that if $d_{k}$ is defined by

$$
d_{k}=\sup _{A \in M_{k}} \min _{x \in A} F^{*}(x)=1 / C_{k},
$$

where $C_{k}$ is associated to $J^{*}$ by (3.2), one has

$$
\lim _{k>+\infty} \searrow d_{k}=0 \text {. }
$$


This last fact is established in Krasnosel'skii [17, Chapter VI] by quite a different proof from the one we present below. So, Theorem 3.3 can be viewed as an extension of this result.

Actually, if the nature of the result in Theorem 3.3 is by no means new, the hypotheses, however, are more general (and simpler) than the ones usually made. We also present a very simple proof that seems to be new.

Proof of TheOREM 3.3. $H$ being separable, let $H_{1} \subset H_{2} \subset \cdots \subset H_{k} \subset \cdots \subset$ $H$ be a nested sequence of finite-dimensional subspaces (dim $\left.H_{k}=k\right)$ spanning $H$ : $\cup_{k=1}^{+\infty} H_{k}=H$. Now observe that for any $A \in M_{k}$, one has $A \cap H_{k}{ }^{\perp} \neq \varnothing$. Indeed, if $A \cap H_{k}{ }^{\perp}=\varnothing$, then denoting by $p_{k}$ the orthogonal projection from $H$ onto $H_{k}$, the mapping $\tau: A \rightarrow S^{k-1}$ defined by $\tau(x)=p_{k} x /\left\|p_{k} x\right\|, x \in A$, is odd and continuous (since in this case $p_{k} x \neq 0 \forall x \in A$ ). But since $A \in M_{k}, A=g\left(S^{k}\right)$ with $g$ odd and continuous, and the mapping $\tau \circ g: S^{k} \rightarrow S^{k-1}$ would be odd and continuous, which is impossible by the Borsuk-Ulam theorem. Thus $A \cap H_{k}^{\perp} \neq \varnothing$.

Now, for any $k \in \mathrm{N}$, let $A_{k} \in M_{k}$ be such that $\max _{u \in A_{k}} J^{*}(u) \leqslant C_{k}+1$. Let $x_{k} \in A_{k} \cap H_{k}^{\perp}$. Thus $J^{*}\left(x_{k}\right) \leqslant C_{k}+1$. Since $H$ is separable, $\left\|x_{k}\right\|=1$ and $x_{k} \in$ $H_{k}^{\perp}$, one has $x_{k} \rightarrow 0$ (weakly in $H$ ). Therefore, $\lim _{k \rightarrow+\infty} J^{*}\left(x_{k}\right)=+\infty$ which implies $\lim _{k \rightarrow+\infty} C_{k}=+\infty$. The proof of Theorem 3.3 is thereby complete.

REMARK 3.5. The preceding argument provides a lower bound for $C_{k}$. Indeed, we have seen above that $\forall A \in M_{k}, A \cap H_{k}{ }^{\perp} \neq \varnothing$. Therefore one has

$$
C_{k} \geqslant \inf _{x \in H_{k}^{\perp} \cap S} J^{*}(x) \text {. }
$$

This estimate will prove to be useful later on.

We are now ready to state the principle of the perturbation method-which is quite simple-in the form of the following result.

THEOREM 3.6. Let $J \in C^{2}(S, \mathbf{R}), J^{*} \in C^{1}(S, \mathbf{R})$ be two functionals satisfying condition (P.S). Assume furthermore that $J^{*}$ is even and bounded from below on $S$. Let $C_{k}, k \in \mathbf{N}$, denote the critical values of $J^{*}$ defined by (3.2). Suppose there exist $k \in \mathbf{N}, \varepsilon>0$ and $a \in \mathbf{R}$ such that $J_{C_{k}+\varepsilon}^{*} \subset J_{a} \subset J_{C_{k+1}-\varepsilon}^{*}$. Then $J$ has at least one critical value in $[a,+\infty)$.

Proof. If $J$ has no critical value in $[a,+\infty)$, then by Theorem 2.1, $J_{a}$ is contractible in itself to a point. Therefore $J_{C_{k}+\varepsilon}^{*}$ is contractible to a point in $J_{C_{k+1}-\varepsilon}^{*}$ which is impossible by Theorem 3.1(iii).

REMARK 3.7. The preceding result remains valid if one only assumes that $J \in C^{0}(S, \mathbf{R}), J \in C^{2}\left(\tilde{J}_{a^{\prime}}, \mathbf{R}\right)$ and that $J$ satisfies condition (P.S) $)_{a^{\prime}}$ for some $a^{\prime}<a$. (See Remark 2.4 above.)

We now examine the analogous result if $J$ has only $C^{1}$ regularity rather than $C^{2}$.

TheOREM 3.8. Let $J, J^{*} \in C^{0}(S, \mathbf{R})$ be two functionals such that $J \in C^{1}\left(\tilde{J}_{a}, \mathbf{R}\right)$, $J^{*} \in C^{1}\left(\tilde{J}_{c}^{*}, \mathbf{R}\right), J$ satisfying condition (P.S $)_{a}$ and $J^{*}$ satisfying condition (P.S) ${ }_{c}$. Assume furthermore that $J^{*}$ is even and bounded from below on $S$. Let $C_{k}$ denote the critical numbers associated with $J^{*}$ defined in (3.2). Suppose there exist $k \in \mathbf{N}, \varepsilon>0$ and $a \in \mathbf{R}$ such that $c \leqslant C_{k}$ and $J_{C_{k}+\varepsilon}^{*} \subset J_{a} \subset J_{a+\varepsilon} \subset J_{C_{k+1}^{*} e^{*}}^{*}$. Then $J$ has at least one critical value in $[a,+\infty)$. 
The proof is as before from Theorems 2.8(iii) and 2.5, since in this case $J_{a}$ is not contractible to a point in $J_{a+e}$.

One also has the following more precise result.

THEOREM 3.9. Under the same hypotheses as in Theorem 3.3 suppose furthermore that $J$ has the property that for any sequence $\left(x_{n}\right) \subset S, J\left(x_{n}\right) \rightarrow+\infty$ if and only if $x_{n} \rightarrow 0$ weakly in $H$. Then, if $J_{C_{k}+\varepsilon}^{*} \subset J_{a} \subset J_{a+\varepsilon} \subset J_{C_{k+1}-\varepsilon}^{*}, J$ has at least one critical value in $[a, \mu(a)]$ where $\mu(a)$ is given by Theorem 2.9 .

The proof is as before but instead of Theorem 2.5, we use Theorems 2.8 and 2.9.

REMARK 3.10. Theorems 3.6, 3.8 and 3.9 are in a sense perturbation results. They somewhat show that the $C_{k}$ are "stable" critical values. If the (noneven) functional $J$ is only a small perturbation of the even functional $J^{*}$, one expects the level sets of $J$ and $J^{*}$ not to be too far apart from each other. Then, if the $C_{k}$ grow fast enough (compare Theorem 3.3) one may hope to verify the conditions of Theorems 3.6 or 3.9. Therefore, in the applications of these theorems, there are clearly two tasks to perform which are:

(i) estimating (from above) the difference $\left|J(x)-J^{*}(x)\right|$,

(ii) estimating (from below) the growth of $C_{k}$ as $k \nearrow+\infty$.

The estimate (3.3) will be helpful in handling the second question.

REMARK 3.11. We would like to make some further comment from a topological viewpoint on the results of Theorems 3.6 and 3.8. Let us first recall a result of Conner and Floyd [11]. For a symmetric and closed subset $K \subset S$, define $\gamma^{+}(K)$ $=\operatorname{Sup}\left\{j \in \mathbf{N} ; \exists h: S^{j} \rightarrow K, h\right.$ odd and continuous $\}\left(\gamma^{+}(K)\right.$ is possibly $\left.+\infty\right)$. Suppose that $\gamma^{+}(K)<+\infty$. Then, by a result of Conner and Floyd [11], any odd and continuous map $h: S^{\gamma^{+}(K)} \rightarrow K$ is essential. That is, if $y_{0} \in h\left(S^{\gamma^{+}(K)}\right)$, then the homotopy class of $h$ in $\Pi_{\gamma^{+}(K)}\left(K, y_{0}\right)^{2}$ is not null (and hence $\Pi_{\gamma^{+}(K)}\left(K, y_{0}\right)$ is not trivial).

Consider again the situation of this section. First observe that if $A \in M_{k}$, then $\gamma^{+}(A) \geqslant k$. Now suppose that for some $k \in \mathbf{N}$, the critical numbers defined in (3.2) are such that $C_{k}<C_{k+1}$. Let $a$ be such that $C_{k}<a<C_{k+1}$. Then, we claim that $\gamma^{+}\left(J_{a}^{*}\right)=k$. Indeed, there exists $A \in M_{k}$ such that $A \subset J_{a}$ (since $a>C_{k}$ ), thus $\gamma^{+}\left(J_{a}^{*}\right) \geqslant k$. On the other hand, if $\gamma^{+}\left(J_{a}\right)>k$, then there exists $B \in M_{k+1}$ such that $B \subset J_{a}^{*}$, which is impossible as $a<C_{k+1}$. This shows at once that for any set $W$ and $\varepsilon>0$ such that $J_{C_{k}+\varepsilon}^{*} \subset W \subset J_{C_{k+1}-\varepsilon}^{*}$, then necessarily $W$ is not contractible in itself to a point. Otherwise, considering an odd and continuous map $h: S^{k} \rightarrow J_{C_{k}+\varepsilon}^{*}$ (such a map exists by the definition of $C_{k}$ ), if $W$ would be contractible in itself to a point, the map $h$ would be null homotopic in $J_{C_{k+1}-e}$, and this would contradict the preceding result of Conner and Floyd as $\gamma^{+}\left(J_{C_{k+1}^{*}-e}^{*}\right)=$ $k$.

This argument provides alternative proofs for Theorems 3.6 and 3.8 via Theorems 2.1 and 2.5. In a sense, it also exhibits a kind of relationship between

\footnotetext{
${ }^{2} \mathrm{By} \Pi_{j}\left(K, y_{0}\right)$ we denote as usual the $j$ th homotopy group of the pair $\left(K, y_{0}\right)$ (that is the $j$ th homotopy group of $K$ computed at the point $y_{0}$ ).
} 
Krasnosel'skii's lemma (Lemma 3.2 here) and the result of Conner and Floyd [11] that we have recalled.

Lastly, let us indicate that the characterization of the existence of critical values by means of changes in the topology of the level sets of a functional (which is exactly the content of a stability result like Theorems 3.6 or 3.8) has been repeatedly emphasized in the works of R. S. Palais [19], [20]. This stability in the change of topology, but in a different context from ours, has also been studied by Marino and Prodi [18].

The remainder of the paper is concerned with applying Theorems 3.6, 3.8 and 3.9 to some nonlinear elliptic problems. In the next two sections, we derive Theorem 1.1 .

4. A functional on the sphere associated with (1.1). Henceforth we will be working in the space $H=H_{0}^{1}(\Omega)$ endowed with the usual norm

$$
\|u\|^{2}=\int_{\Omega}|\nabla u|^{2} d x
$$

and scalar product

$$
(u, v)=\int_{\Omega} \nabla u \cdot \nabla v d x
$$

$S=\left\{u \in H_{0}^{1}(\Omega) ;\|u\|=1\right\}$. We denote by $\langle$,$\rangle the duality of either H^{-1}(\Omega)$ and $H_{0}^{1}(\Omega)$ or of $T_{u} S^{\prime}$ and $T_{u} S \simeq\{u\}^{\perp}$. We recall that

$$
\begin{aligned}
I(u) & =\frac{1}{2} \int_{\Omega}|\nabla u|^{2} d x-\frac{1}{p+1} \int_{\Omega}|u|^{p+1} d x-\int_{\Omega} h u d x, \\
I^{*}(u) & =\frac{1}{2} \int_{\Omega}|\nabla u|^{2} d x-\frac{1}{p+1} \int_{\Omega}|u|^{p+1} d x .
\end{aligned}
$$

( $I^{*}$ is the even part of $I$.) Associated with $I$ (resp. $I^{*}$ ), we define a functional $J$ (resp. $J^{*}$ ) on $S$ such that the "free" critical points of $I$, that is the solutions of (1.1), which correspond to positive critical values, are derived from the "constrained" critical points of $J$, and similarly for $I^{*}$ and $J^{*}$. We set

$$
\begin{cases}J(u)=\max _{\lambda>0} I(\lambda u), & u \in S, \\ J^{*}(u)=\max _{\lambda>0} I^{*}(\lambda u), & u \in S .\end{cases}
$$

We always assume in the following that

$$
1<p<(N+2) /(N-2) \text { if } N \geqslant 3 \text { and } 1<p<+\infty \text { if } N=2 \text {. }
$$

Proposition 4.1. Under condition (4.2), for any $h \in L^{2}(\Omega), J \in C^{0}(S, \mathbf{R}), J>0$, $J \in C^{2}\left(\tilde{J}_{\varepsilon}, \mathbf{R}\right)$ and $J$ satisfies conditions (P.S $)_{\varepsilon}$ for any $\varepsilon>0$. Furthermore, $J^{*}$ is even and verifies the condition in Theorem 3.3.

Proposition 4.2. For any $h \in L^{2}(\Omega)$ and any $u \in S$ such that $J(u)>0$, there exists a unique $\lambda=\lambda(u)>0$ such that $J(u)=I(\lambda(u) u)$. Then, one has

$$
\left\langle I^{\prime}(\lambda(u) u), u\right\rangle=0 \text { and }\left\langle I^{\prime}(\lambda(u) u), \phi\right\rangle=\lambda(u)^{-1}\left\langle J^{\prime}(u), \phi\right\rangle
$$

for any $\phi \in T_{u} S \simeq\{u\}^{\perp}$. Furthermore, the positive critical values of $I$ (defined in $H_{0}^{1}(\Omega)$ ) and of $J$ (defined on $S$ ) are the same. 
Proofs of Propositions 4.1 AND 4.2. One has for $u \in S$,

$$
I(\lambda u)=\frac{\lambda^{2}}{2}-\frac{\lambda^{p+1}}{p+1} \int_{\Omega}|u|^{p+1}-\lambda \int_{\Omega} h u .
$$

(From now on, the Lebesgue measure $d x$ is understood in all integrals.) Since $u \in S$, one has $\int_{\Omega}|u|^{p+1}>0$ and it is clear from (4.3) that $J$ is well defined (i.e. finite) for all $u \in S$, is nonnegative, and that $J$ is continuous on $S$ (since $I$ is continuous and $C^{1}$ for the $H_{0}^{1}(\Omega)$ topology, given the limitation imposed upon $p$ in (4.2)). Let us first show that $\lambda=\lambda(u)>0$ is uniquely determined from the condition $J(u)=I(\lambda u)$ when $\lambda>0$. Differentiating (4.3) yields

$$
\left\{\begin{array}{l}
\frac{d}{d \lambda} I(\lambda u)=\lambda-\lambda^{p} \int_{\Omega}|u|^{p+1}-\int_{\Omega} h u, \\
\frac{d^{2}}{d \lambda^{2}} I(\lambda u)=1-p \lambda^{p-1} \int_{\Omega}|u|^{p+1} .
\end{array}\right.
$$

Thus, there exists $\lambda_{0}(u)\left(=\left\{p \int_{\Omega}|u|^{p+1}\right\}^{-1 /(p-1)}\right)$ such that $d^{2} I(\lambda u) / d \lambda^{2}$ is positive for $0<\lambda<\lambda_{0}(u)$ and is negative for $\lambda>\lambda_{0}(u)$. Hence, there are two cases:

(i) If $(d I(\lambda u) / d \lambda)_{\mid \lambda=0}=-\int_{\Omega} h u \geqslant 0$, then $d I(\lambda u) / d \lambda$ vanishes only once at a value $\lambda=\lambda(u)>0$ such that $\lambda \mapsto I(\lambda u)$ achieves its maximum.

(ii) If $(d I(\lambda u) / d \lambda)_{\mid \lambda=0}<0$, and if $J(u)>0$, then $d I(\lambda u) / d \lambda$ vanishes twice, once at a value of $\lambda$ that produces a local negative minimum of $I(\lambda u)$ and once more at a value $\lambda=\lambda(u)$ that yields the maximum of $I(\lambda u)$.

Therefore, in both cases $\lambda=\lambda(u)$ such that $J(u)=I(\lambda u)>0$ is seen to be unique. Furthermore, $\lambda=\lambda(u)$ is uniquely determined from the conditions

$$
\left\{\begin{array}{l}
I(\lambda u)>0 \\
d I(\lambda u) / d \lambda=\left\langle I^{\prime}(\lambda u), u\right\rangle=0 \\
d^{2} I(\lambda u) / d \lambda^{2}=I^{\prime \prime}(\lambda u)(u, u)<0 .
\end{array}\right.
$$

It is now clear from (4.5), using the implicit function theorem, that $u \rightarrow \lambda(u)$ is a $C^{1}$ function on $\tilde{J}_{\varepsilon}$ (for any $\varepsilon>0$ ). Whence, $J(u)=I(\lambda(u) u)$ is certainly $C^{1}$ on $\tilde{J}_{\varepsilon}$. Observe moreover that for any $\phi \in T_{u} S$ one has

$$
\left\langle J^{\prime}(u), \phi\right\rangle=\lambda(u)\left\langle I^{\prime}(\lambda(u) u), \phi\right\rangle+\left\langle\lambda^{\prime}(u), \phi\right\rangle\left\langle I^{\prime}(\lambda(u) u), u\right\rangle .
$$

But since $\left\langle I^{\prime}(\lambda(u) u), u\right\rangle=0$, one derives

$$
\left\langle J^{\prime}(u), \phi\right\rangle=\lambda(u)\left\langle I^{\prime}(\lambda(u) u), \phi\right\rangle, \quad \forall \phi \in T_{u} S .
$$

Since $I$ is of class $C^{2}$ and $\lambda$ of class $C^{1}$ on $\tilde{J}_{\varepsilon}$, it is seen at once from (4.6) (which does not involve $\lambda^{\prime}(u)$ any longer) that $J$ is actually of class $C^{2}$ on $\tilde{J}_{e}$, for any $\varepsilon>0$.

Thus, if $u \in S$ is such that $J(u)>0$ and $J^{\prime}(u)=0$, one has $I^{\prime}(\lambda(u) u)=0$ (indeed $I^{\prime}(\lambda(u) u)$ vanishes on both $\mathbf{R}\{u\}$ and $\left.\{u\}^{\perp}\right)$. Conversely, if $v \in H_{0}^{1}(\Omega)$ is such that $I(v)>0$ and $I^{\prime}(v)=0$, then, setting $u=v /\|v\| \in S$, one has $J(u)=$ $I(v)$ and $J^{\prime}(u)=0$ (recall that $\lambda \mapsto I(\lambda u)$ has at most only one positive "extremal"). The positive critical values of $J$ and $I$ are therefore the same.

Lastly, let us show that $J$ verifies condition (P.S) $)_{\varepsilon}$. Let $C \geqslant \varepsilon$ and $\left(u_{n}\right) \subset S$ be such that $\varepsilon \leqslant J\left(u_{n}\right) \leqslant C$ and $J^{\prime}\left(u_{n}\right) \rightarrow 0$. We wish to show that $\left(u_{n}\right)$ has a convergent subsequence. Set $v_{n}=\lambda\left(u_{n}\right) u_{n}$. We claim that $\left\|I^{\prime}\left(v_{n}\right)\right\|_{H^{-1}(\Omega)}<C_{1}\left\|J^{\prime}\left(u_{n}\right)\right\|_{T_{u_{n}}^{\prime} s}$ 
for some constant $C_{1}$ (depending on $\varepsilon$ ). Indeed, decompose $H_{0}^{1}(\Omega)=\mathbf{R}\left\{u_{n}\right\} \perp T_{u_{n}} S$ so that $\forall \psi \in H_{0}^{1}(\Omega), \psi=t u_{n}+\phi$ with $t \in \mathbf{R},\left(u_{n}, \phi\right)=0$ and $\|\psi\|_{H_{0}^{1}}^{2}=t^{2{ }^{2}}+$ $\|\phi\|_{T_{u_{n}} s}^{2}$. Now, observe that since $\left\langle I^{\prime}\left(v_{n}\right), u_{n}\right\rangle=0$, one has $\left\langle I^{\prime}\left(v_{n}\right), \psi\right\rangle=$ $\left\langle I^{\prime}\left(v_{n}\right), \phi\right\rangle=\lambda\left(u_{n}\right)^{-1}\left\langle J^{\prime}\left(u_{n}\right), \phi\right\rangle$. Thus,

$$
\left\|I^{\prime}\left(v_{n}\right)\right\|_{H^{-1}(\Omega)}=\sup _{\substack{\psi \in H_{0}^{1}(\Omega) \\\|\psi\|<1}}\left\langle I^{\prime}\left(v_{n}\right), \psi\right\rangle \leqslant \lambda\left(u_{n}\right)^{-1}\left\|J^{\prime}\left(u_{n}\right)\right\|_{T_{u_{n}}^{\prime} s .}
$$

The sequence $\lambda\left(u_{n}\right)^{-1}$ is bounded from above; $\lambda\left(u_{n}\right)^{-1} \leqslant C_{1}$. Indeed, if not there would be a subsequence, denoted again $\lambda\left(u_{n}\right)$, such that $\lambda\left(u_{n}\right) \rightarrow 0$. But this would imply $J\left(u_{n}\right)=I\left(\lambda\left(u_{n}\right) u_{n}\right) \rightarrow 0$, which is impossible since $J\left(u_{n}\right) \geqslant \varepsilon>0$. (This step in the proof explains why we use condition (P.S) $)_{\varepsilon}$. Note that $J$ does not satisfy (P.S) $)_{0}$ ) From (4.7) we derive

$$
\left\|I^{\prime}\left(v_{n}\right)\right\|_{H^{-1}(\Omega)} \leqslant C_{1}\left\|J^{\prime}\left(u_{n}\right)\right\|_{T_{u_{n}}^{\prime} s} .
$$

Thus, the sequence $\left(v_{n}\right) \subset H_{0}^{1}(\Omega)$ satisfies $\varepsilon \leqslant I\left(v_{n}\right) \leqslant C$ and $I^{\prime}\left(v_{n}\right) \rightarrow 0$ in $H^{-1}(\Omega)$. It is well known (see, e.g., Rabinowitz [21]) that $I$ satisfies the (P.S) condition in the space $H_{0}^{1}(\Omega)$. Therefore, one can extract from $\left(v_{n}\right)$ a convergent subsequence. For the sake of completeness, we repeat the proof of this fact here. Let

$$
\varepsilon_{n}=-\Delta v_{n}-\left|v_{n}\right|^{p-1} v_{n}-h(x)
$$

so that $\left\|\varepsilon_{n}\right\|_{H^{-1}(\Omega)} \rightarrow 0$. Thus,

$$
\left.\left|\int_{\Omega}\right| \nabla v_{n}\right|^{2}-\int_{\Omega}\left|v_{n}\right|^{p+1}-\int_{\Omega} h v_{n}\left|\leqslant\left\|\varepsilon_{n}\right\|_{H^{-1}(\Omega)}\right|\left\|v_{n}\right\|_{H_{0}^{\prime}(\Omega)} .
$$

$v_{n}$ also satisfies

$$
\varepsilon \leqslant \frac{1}{2} \int_{\Omega}\left|\nabla v_{n}\right|^{2}-\frac{1}{p+1} \int_{\Omega}\left|v_{n}\right|^{p+1}-\int_{\Omega} h v_{n} \leqslant C .
$$

We denote thereafter by $C$ various positive constants. Combining (4.9) with (4.10) and the fact that $\left|\int_{\Omega} h v_{n}\right| \leqslant\|h\|_{H^{-1}(\Omega)}\left\|v_{n}\right\|_{H_{0}^{1}(\Omega)}$, one derives that

$$
\left(\frac{1}{2}-1 /(p+1)\right)\left\|v_{n}\right\|_{H_{0}^{1}}^{2} \leqslant C\left\|v_{n}\right\|_{H_{0}^{1}}+C,
$$

from where it follows that $\left\|v_{n}\right\|_{H_{0}^{1}} \leqslant C$. By (4.2), the injection $H_{0}^{1}(\Omega) \hookrightarrow L^{p+1}(\Omega)$ is compact (Sobolev injection theorem). Therefore, there exists a subsequence of $v_{n}$, denoted again by $v_{n}$, which converges strongly in $L^{p+1}(\Omega)$. Thus, $\left|v_{n}\right|^{p-1} v_{n}$ converges strongly in $L^{(p+1) / p}$ and also in $H^{-1}(\Omega)$ (since by $(4.2), L^{(p+1) / p}(\Omega) \subset$ $H^{-1}(\Omega)$ ). From (4.8) we then derive that $v_{n}$ converges strongly in $H_{0}^{1}(\Omega)$. Thus, we have proved the claim that $I$ satisfies condition (P.S) in $H_{0}^{1}(\Omega)$.

Turning back to the functional $J$ and to $u_{n}$, we have $u_{n}=v_{n} /\left\|v_{n}\right\|$. Observe that since $v_{n} \rightarrow v$ in $H_{0}^{1}(\Omega)$, then $I\left(v_{n}\right) \rightarrow I(v)$. As $I\left(v_{n}\right) \geqslant \varepsilon$, this implies $I(v) \geqslant \varepsilon>0$ and $v \neq 0$. Therefore, $u_{n}$ converges strongly in $S$ (i.e. for the $H_{0}^{1}(\Omega)$ topology on $S$ ). This concludes the proof that $J$ satisfies the (P.S) $)_{e}$ condition.

The proofs of Propositions 4.1 and 4.2 are now complete except for the fact that $J^{*}$ verifies the condition in Theorem 3.3 which is included in the following more general and more precise estimates. (Indeed $J^{*}$ is but a particular case of $J$ by taking $h=0$.) 
LEMMA 4.3. Let $h \in L^{2}(\Omega)$ be given and $p>1$ satisfy (4.2). Then for any sequence $\left(u_{n}\right) \subset S$, the following are equivalent:

(i) $J\left(u_{n}\right) \rightarrow+\infty$,

(ii) $\lambda\left(u_{n}\right) \rightarrow+\infty$,

(iii) $u_{n} \rightarrow 0$ strongly in $L^{p+1}(\Omega)$ (or, equivalently,

(iv) $u_{n} \rightarrow 0$ weakly in $\left.H_{0}^{1}(\Omega)\right)$.

Furthermore, for any sequence $\left(u_{n}\right) \subset S$ such that $J\left(u_{n}\right) \rightarrow+\infty$, one has ${ }^{3}$

$$
\lambda\left(u_{n}\right) \sim \lambda^{*}\left(u_{n}\right)=\left(\int_{\Omega}\left|u_{n}\right|^{p+1}\right)^{-1 /(p-1)}
$$

and

$$
J\left(u_{n}\right) \sim J^{*}\left(u_{n}\right)=\left(\frac{1}{2}-\frac{1}{p+1}\right)\left(\int_{\Omega}\left|u_{n}\right|^{p+1}\right)^{-2 /(p-1)} .
$$

Proof. Let $\left(u_{n}\right) \subset S$ be a sequence such that $J\left(u_{n}\right) \rightarrow+\infty$. Since $J\left(u_{n}\right)=$ $I\left(\lambda\left(u_{n}\right) u_{n}\right)$ and $I$ is bounded on bounded sets of $H_{0}^{1}(\Omega)$, it is clear that $\lambda\left(u_{n}\right) \rightarrow+\infty$. From (4.4) and (4.5) we know that $\lambda_{n}=\lambda\left(u_{n}\right)$ satisfies

$$
\begin{gathered}
1<p \lambda_{n}^{p-1} \int_{\Omega}\left|u_{n}\right|^{p+1} \\
1-\lambda_{n}^{p-1} \int_{\Omega}\left|u_{n}\right|^{p+1}-\lambda_{n}^{-1} \int_{\Omega} h u_{n}=0 \\
J\left(u_{n}\right)=\lambda_{n}^{2}\left\{\frac{1}{2}-\frac{1}{p+1} \lambda_{n}^{p-1} \int_{\Omega}\left|u_{n}\right|^{p+1}-\lambda_{n}^{-1} \int_{\Omega} h u_{n}\right\}
\end{gathered}
$$

Hence, $u_{n} \rightarrow 0$ strongly in $L^{p+1}(\Omega)$ if $\lambda_{n} \rightarrow+\infty$. Since the weak topology on $S$ is metrizable $\left(H_{0}^{1}(\Omega)\right.$ being separable) and the injection $H_{0}^{1}(\Omega) \hookrightarrow L^{p+1}(\Omega)$ is compact, $u_{n} \rightarrow 0$ strongly in $L^{p+1}(\Omega)$ is equivalent to $u_{n} \rightarrow 0$ weakly in $H_{0}^{1}(\Omega)$ for $\left(u_{n}\right) \subset S$. Conversely, suppose $u_{n} \rightarrow 0$ strongly in $L^{p+1}(\Omega)$, then, by (4.13) $\lambda_{n} \rightarrow+\infty$. So far, we have shown that (i) $\Rightarrow$ (ii) $\Leftrightarrow$ (iii) $\Leftrightarrow$ (iv).

Now, if $\lambda_{n} \rightarrow+\infty$, it follows from (4.14) (since $\int_{\Omega} h u_{n}$ is bounded) that

$$
\lim \lambda_{n}^{p-1} \int_{\Omega}\left|u_{n}\right|^{p+1}=1
$$

that is,

$$
\lambda_{n} \sim\left(\int_{\Omega}\left|u_{n}\right|^{p+1}\right)^{-1 /(p-1)}
$$

Furthermore, then,

$$
\lim _{n \rightarrow+\infty} J\left(u_{n}\right) \lambda_{n}^{-2}=\frac{1}{2}-\frac{1}{p+1},
$$

\footnotetext{
${ }^{3}$ In the sequel, the notation $a_{n} \sim b_{n}$ for two sequences $\left(a_{n}\right)$ and $\left(b_{n}\right)$ means as usual that $\lim _{n \rightarrow+\infty} a_{n} b_{n}^{-1}=1$.
} 
which shows firstly that $\lim _{n \rightarrow+\infty} J\left(u_{n}\right)=+\infty$ (hence (ii) $\Rightarrow$ (i)), and also that (by (4.15))

$$
J\left(u_{n}\right) \sim\left(\frac{1}{2}-\frac{1}{p+1}\right)\left(\int_{\Omega}\left|u_{n}\right|^{p+1}\right)^{-2 /(p-1)} .
$$

Lastly, denoting by $\lambda^{*}(u)$ the unique positive number such that $J^{*}(u)=I^{*}\left(\lambda^{*}(u) u\right)$ $>0$, it is immediate from (4.14) and (4.15) that when $h \equiv 0$, that is for the case of $J^{*}$, one then exactly has

$$
\lambda^{*}(u)=\left(\int_{\Omega}|u|^{p+1}\right)^{-1 /(p-1)}
$$

and

$$
J^{*}(u)=\left(\frac{1}{2}-\frac{1}{p+1}\right)\left(\int_{\Omega}|u|^{p+1}\right)^{-2 /(p-1)} .
$$

This completes the proof of Lemma 4.3.

In the next step, we derive an estimate for $\left|J(u)-J^{*}(u)\right|$.

LEMMA 4.4. There exists a constant $\gamma>0$ such that for any $u \in S$ with $J(u) \geq 1$ and $J^{*}(u) \geqslant 1$, one has

$$
\left\{\begin{array}{l}
\left|J(u)-J^{*}(u)\right| \leqslant \gamma[J(u)]^{1 /(p+1)} \\
\left|J(u)-J^{*}(u)\right| \leqslant \gamma\left[J^{*}(u)\right]^{1 /(p+1)}
\end{array}\right.
$$

Proof. We denote $\lambda=\lambda(u)$ and $\lambda^{*}=\lambda^{*}(u)$. Clearly,

$$
\begin{gathered}
J(u)-J^{*}(u) \leqslant I(\lambda u)-I^{*}(\lambda u)=-\lambda \int_{\Omega} h u, \\
J^{*}(u)-J(u) \leqslant I^{*}\left(\lambda^{*} u\right)-I\left(\lambda^{*} u\right)=\lambda^{*} \int_{\Omega} h u .
\end{gathered}
$$

Whence,

$$
\left|J(u)-J^{*}(u)\right| \leqslant\left\{\max \left(\lambda, \lambda^{*}\right)\right\}\|u\|_{L^{p+1}(\Omega)}\|h\|_{L^{q}(\Omega)}
$$

where $1 /(p+1)+1 / q=1$. Now, to derive for instance the first estimate in (4.18), we argue by contradiction. We assume that there exists a sequence $\left(u_{n}\right) \subset S$ such that $J\left(u_{n}\right) \geqslant 1$ and $\tilde{J}\left(u_{n}\right) \geqslant 1$ while

$$
\left|J\left(u_{n}\right)-J^{*}\left(u_{n}\right)\right| \geqslant n\left[J\left(u_{n}\right)\right]^{1 /(p+1)}, \quad \forall n \in \mathbf{N} .
$$

Then, $\left|J\left(u_{n}\right)-J^{*}\left(u_{n}\right)\right| \rightarrow+\infty$, and comparing with (4.19) it follows that $\left\|u_{n}\right\|_{L^{p+1}(\Omega)}$ $\rightarrow 0$. (For if not, along a subsequence where $\|u\|_{L^{p+1}(\Omega)}$ is bounded away from zero, $\lambda(u)$ and $\lambda^{*}(u)$ would be bounded by Lemma 4.3, whence, by (4.19), $\left|J(u)-J^{*}(u)\right|$ would be bounded.) But then, we derive from (4.11) and (4.19) that

$$
\left|J\left(u_{n}\right)-J^{*}\left(u_{n}\right)\right| \leqslant A_{n}
$$

with

$$
A_{n} \sim\|h\|_{L^{q}(\Omega)}\left\|u_{n}\right\|_{L^{p+1}(\Omega)}^{-2 /(p-1)} .
$$


On the other hand, $A_{n}$ should satisfy $A_{n} \geqslant n\left[J\left(u_{n}\right)\right]^{1 /(p+1)}$, and since $u_{n} \rightarrow 0$ strongly in $L^{p+1}(\Omega)$, we know from Lemma 4.3 that $J\left(u_{n}\right) \rightarrow+\infty$ and

$$
J\left(u_{n}\right)^{1 /(p+1)} \sim C\left\|u_{n}\right\|_{L^{p+1}}^{-2 /(p-1)}
$$

where $C>0$ is a constant. This is contradictory and therefore (4.20) is impossible.

The proof of the second estimate in $(4.18)$ is very much the same.

REMARK 4.5. The idea of looking for critical points of $J$ on the sphere $S$ in order to solve (1.1) is related to a method introduced by Coffman [10] and Hempel [16]. In the odd case (i.e. when $h \equiv 0$ ), to show the existence of infinitely many solutions of (1.1), or, equivalently, to find critical points of $I^{*}$, they look for "constrained" critical points of the trace of $I^{*}$ on the manifold.

$$
\Re^{*}=\left\{v \in H_{0}^{1}(\Omega) ; v \neq 0 \text { and }\left\langle I^{* \prime}(v), v\right\rangle=0\right\} \text {. }
$$

Indeed, clearly, any nontrivial critical point of $I^{*}$ is to be sought on this manifold. In this case, since $\lambda^{*}(u)$ is uniquely determined from the relation $\left\langle I^{* \prime}\left(\lambda^{*}(u) u\right), u\right\rangle$ $=0$ for any $u \in S$, one has

$$
\mathfrak{T}^{*}=\left\{v=\lambda^{*}(u) u ; u \in S\right\},
$$

and $\mathfrak{R}^{*}$ is but the image of $S$ under the mapping $u \rightarrow\|u\|_{L^{p}+p_{1}^{+1)}}^{-(p-1)} u$.

In the general case (i.e. $h \neq 0$ ), defining $\mathscr{N}=\left\{v \in H_{0}^{1}(\Omega) ; v \neq 0,\left\langle I^{\prime}(v), v\right\rangle=\right.$ $0\}$, then $\mathfrak{N}$ is not exactly the image of $S$. Nevertheless, the submanifold $\mathfrak{K}_{\varepsilon}=\mathfrak{R}$ $\cap\{v ; I(v) \geqslant \varepsilon\}$ is again the image of $\tilde{J}_{\varepsilon}$ under the mapping $u \mapsto \lambda(u) u$ for any $\varepsilon>0$. However, we find it more convenient, having in mind the results of $\S 3$, to work on $S$ which is a symmetric or a fixed point free involution manifold rather than on $\mathfrak{N}$ (or $\mathfrak{K}_{\mathfrak{e}}$ ) which are not.

5. Proof of Theorem 1.1. The results of $\S 3$ will now be applied to the functionals $J$ and $J^{*}$ on $S$ introduced in the preceding section. Observe that $J$ and $J^{*}$ satisfy the hypotheses of Theorem 3.3. We recall that the $C_{k}(k \in \mathrm{N})$ are the critical values of $J^{*}$ defined by (3.2). We first require an estimate from below on the growth of $C_{k}$ as $k \rightarrow+\infty$.

Proposition 5.1. Assume (4.2). There exists a constant $C>0$ (respectively, there exists, for any $\varepsilon>0$ a constant $C_{\varepsilon}>0$ ) such that:

$$
\begin{cases}C_{k} \geqslant C k^{\alpha_{N}} & \text { if } N \geqslant 3, \forall k \in \mathbf{N}, \\ C_{k} \geqslant C_{\varepsilon} k^{\alpha_{2}-\varepsilon} & \text { if } N=2, \forall k \in \mathbf{N},\end{cases}
$$

where

$$
\alpha_{N}=\frac{2 N-(N-2)(p+1)}{N(p-1)}, \quad \forall N>2 .
$$

Proof. Let $\lambda_{k}$ be the $k$ th eigenvalue (counting multiplicities) of $-\Delta: H_{0}^{1}(\Omega) \rightarrow$ $H^{-1}(\Omega)$. Let $\left\{\phi_{1}, \ldots, \phi_{k}, \ldots\right\}$ denote an orthonormalized set of eigenfunctions of $-\Delta$ in $H_{0}^{1}(\Omega)$ such that $\phi_{k}$ is associated with $\lambda_{k}:-\Delta \phi_{k}=\lambda_{k} \phi_{k}$. Let $H_{k}$ be the $k$-dimensional subspace of $H_{0}^{1}(\Omega)$ spanned by $\left\{\phi_{1}, \ldots, \phi_{k}\right\}$. We recall from $\S 3$ that

$$
C_{k} \geqslant \inf _{u \in H_{k}^{\perp} \cap S} J^{*}(u)
$$


For any $u \in H_{k}{ }^{\perp} \cap S$, one has $\int_{\Omega}|\nabla u|^{2}=1$ and $\int_{\Omega} u^{2} \leqslant \lambda_{k}^{-1}$. By Hölder's inequality one has

$$
\|u\|_{L^{p+1}(\Omega)} \leqslant\|u\|_{L^{2}(\Omega)}^{\theta}\|u\|_{L^{2^{*}}(\Omega)}^{1-\theta}
$$

where $2^{*}=2 N /(N-2)$ if $N \geqslant 3$ (whence $2<p+1<2^{*}$ by (4.2)) and $2^{*}$ is any (finite) number larger than $p+1$ if $N=2$. Here, $\theta$ is given by $\theta / 2+(1-\theta) / 2^{*}$ $=1 /(p+1)(0<\theta<1)$. That is, $\theta=N /(p+1)-(N-2) / 2$ if $N \geqslant 3$ and $\theta$ can be made arbitrarily close to (but smaller than) $2 /(p+1)$ by choosing $2^{*}$ arbitrarily large when $N=2$. By Sobolev's imbedding theorem, since $u \in S$, one has $\|u\|_{L^{*}} \leqslant C$ (the constant $C$ depends on the choice of $2^{*}$ in dimension 2).

Thus, for any $u \in H_{k}^{\perp} \cap S$, one has

$$
\|u\|_{L^{p+1}(\Omega)} \leqslant C \lambda_{k}^{-\theta / 2}
$$

Hence, for any $u \in H_{k}{ }^{\perp} \cap S$,

$$
J^{*}(u)=C\|u\|_{L^{p+1}(\Omega)}^{-2(p+1) /(p-1)} \geqslant C \lambda_{k}^{\theta(p+1) /(p-1)}
$$

where $C>0$ denotes various constants. Now, recall that by the formula for the asymptotic behaviour of $\lambda_{k}$ as $k \rightarrow+\infty$ (see Agmon [1, \&14] or Courant-Hilbert [12]) one has $\lambda_{k} \sim C k^{2 / N}$. Hence, we derive, using (3.3), that

$$
C_{k} \geqslant C k^{\alpha_{N}} \text { if } N \geqslant 3, \forall k \in \mathbf{N},
$$

where

$$
\alpha_{N}=\theta \frac{2}{N} \frac{p+1}{p-1}=\frac{2 N-(N-2)(p+1)}{N(p-1)} .
$$

In the case of dimension 2, by choosing $2^{*}$ large enough $\theta$ can be made arbitrarily close to $2 /(p+1)$. Thus, when $N=2$, for any $\varepsilon>0$, there exists $C_{\varepsilon}>0$ such that $C_{k} \geqslant C_{\varepsilon} k^{\alpha_{2}-\varepsilon}$ where $\alpha_{2}=2 /(p-1)$. This concludes the proof of Proposition 5.1.

REMARK 5.2. In the case of dimension $N=1$, the above argument would merely show $C_{k} \geqslant C k^{2 /(p-1)}$. Actually, in this case, a more careful estimate of $\|u\|_{L^{p+1}(\Omega)}$ when $u \in H_{k}^{\perp} \cap S$ shows that $C_{k}>C k^{(p+1) /(p-1)}$. An analogous estimate is being derived and employed in our study of the existence of periodic solutions for some forced second order systems of ordinary differential equations in [9]. (See also [8].)

We are now ready to complete the proof of Theorem 1.1. In view of Theorem 3.6 (cf. Remark 3.7 and also Theorem 3.8), it will suffice to show the existence of infinitely many distinct values of $k \in \mathbf{N}$, together with the existence of $\varepsilon_{k}>0$ and $a_{k} \in \mathbf{R}$ such that $J_{C_{k}+\varepsilon_{k}}^{*} \subset J_{a_{k}} \subset J_{C_{k+1}-\varepsilon_{k}}^{*}$. From Lemma 4.3 we derive the existence of $A \geqslant 1$ such that for any $u \in S$ with $J^{*}(u) \geqslant A$ one also has $J(u) \geqslant 1$ and, similarly, $J(u) \geqslant A \Rightarrow J^{*}(u) \geqslant 1$. (Indeed, just argue by contradiction and observe that for any sequence $\left(u_{n}\right) \subset S, J\left(u_{n}\right) \rightarrow+\infty \Leftrightarrow J^{*}\left(u_{n}\right) \rightarrow+\infty$.) Hence, by Lemma 4.4 for any $C \geqslant A$, one has the inclusions

$$
J_{C}^{*} \subset J_{\mu(C)} \subset J_{\mu_{1}(C)}^{*} \text { where } \mu(C)=C+\gamma C^{1 /(p+1)}
$$

and

$$
\mu_{1}(C)=\mu(C)+\gamma[\mu(C)]^{1 /(p+1)}=\mu[\mu(C)]
$$


( $\gamma>0$ is the constant in (4.18) of Lemma 4.4.) Since $C \geqslant 1$ and $C^{1 /(p+1)}<C$, we have $\mu_{1}(C) \leqslant \nu(C)$ with $\nu(C)=C+\delta C^{1 /(p+1)}$ where $\delta=\gamma+\gamma(1+\gamma)^{1 /(p+1)}$. Thus, we just need to show that for infinitely many distinct values of $k$, we have $C_{k+1}>\nu\left(C_{k}\right)$. Indeed, whenever $C_{k+1}>\nu\left(C_{k}\right)$, we can choose $\varepsilon_{k}>0$ small enough so that $C_{k+1}-\varepsilon_{k}>\nu\left(C_{k}+\varepsilon_{k}\right)$ and denoting $a_{k}=\mu\left(C_{k}+\varepsilon_{k}\right)$, we then have

$$
J_{C_{k}+\varepsilon_{k}}^{*} \subset J_{a_{k}} \subset J_{\nu}^{*}\left(C_{k}+\varepsilon_{k}\right) \subset J_{C_{k+1}-\varepsilon_{k}}^{*}
$$

Thus, for any such $k$, there exists a critical value of $J$ in $\left[a_{k},+\infty\right)$. Note that since $a_{k} \geqslant C_{k}$ and $\lim _{k \rightarrow+\infty} C_{k}=+\infty$, the existence of infinitely many such $k \in \mathbf{N}$ implies the existence of infinitely many distinct critical values of $J$ which converge to $+\infty$.

We now argue by contradiction and suppose that there exists $k_{0} \in \mathbf{N}$ such that for any $k \geqslant k_{0}$, one has $C_{k+1} \leqslant \nu\left(C_{k}\right)$, that is:

$$
C_{k+1}-C_{k} \leqslant \delta C_{k}^{1 /(p+1)}, \quad \forall k \geqslant k_{0} .
$$

We require the following simple observation.

LEMMA 5.3. Let $\left(d_{k}\right)_{k \in N}$ be a sequence of positive numbers such that $0 \leqslant d_{k+1}-d_{k}$ $\leqslant \delta d_{k}^{\theta}, \forall k \geqslant k_{0}$, where $k_{0} \in \mathbf{N}, \delta>0$ and $0<\theta<1$. Then there exists a constant $C>0$ such that $d_{k} \leqslant C k^{1 /(1-\theta)}, \forall k \geqslant 1$.

Proof of Lemma 5.3. Set $\delta_{k}=k^{-1 /(1-\theta)} d_{k}>0$. We want to show that $\delta_{k}$ remains bounded as $k \rightarrow+\infty$. Using the fact that $(1+t)^{\beta} \geqslant 1+\beta t$ for $\beta=$ $1 /(1-\theta)>1$ and $t>0$, we derive from $0 \leqslant d_{k+1}-d_{k} \leqslant \delta d_{k}^{\theta}$ that

$$
1 /(1-\theta) \delta_{k+1} k^{-1}+\delta_{k+1}-\delta_{k} \leqslant \delta \delta_{k}^{\theta} k^{-1},
$$

whence, it follows that:

(i) either $\delta_{k+1} \leqslant \delta_{k}$;

(ii) or $\delta_{k} \leqslant \delta_{k+1} \leqslant \delta(1-\theta) \delta_{k}^{\theta}$, which implies $\delta_{k} \leqslant[\delta(1-\theta)]^{1 /(1-\theta)}$ and thus, $\delta_{k+1} \leqslant[\delta(1-\theta)]^{1 /(1-\theta)}=M$.

Hence, we always have $\delta_{k+1} \leqslant \max \left(\delta_{k}, M\right), \forall k \geqslant k_{0}$, from where it follows that $\delta_{k} \leqslant \max \left(\delta_{k_{0}}, M\right)$ and the sequence $\delta_{k}$ is bounded.

Let us continue the proof of Theorem 1.1. Assuming (5.2) we derive from Lemma 5.3 that

$$
C_{k+1} \leqslant C k^{(p+1) / p}, \quad \forall k \geqslant 1 .
$$

Now, recalling Proposition 5.1 we know that

$$
\begin{cases}C_{k} \geqslant C k^{\alpha_{N}} & \text { if } N \geqslant 3, \forall k \geqslant 1, \\ C_{k} \geqslant C_{\varepsilon} k^{\alpha_{2}-\varepsilon} & \text { if } N=2, \forall k \geqslant 1\end{cases}
$$

(with some other positive constants $C, C_{e}$ ). Therefore, comparing (5.1) and (5.3) yields in all cases $\alpha_{N} \leqslant(p+1) / p, \forall N \geqslant 2$. This inequality reads precisely

$$
(2 N-2) p^{2}-(N+2) p-N \geqslant 0 \text {. }
$$

Thus, if $p_{N}$ denotes the largest root of $(2 N-2) p^{2}-(N+2) p-N=0\left(1<p_{N}<\right.$ $(N+2) /(N-2)$ as is easily checked), for any $p$ such that $1<p<p_{N}$, the preceding inequality is violated and therefore (5.2) cannot hold. Thus, there exist infinitely many values of $k \in \mathbf{N}$ such that $J_{C_{k}+\varepsilon_{k}}^{*} \subset J_{a_{k}} \subset J_{C_{k+1}-\varepsilon_{k}}^{*}$. The proof of Theorem 1.1 is now complete. 
REMARK 5.4. In the case of dimension 1, we know that $C_{k} \geqslant C k^{(p+1) /(p-1)}$ (see Remark 5.2 above and [9]). Thus, in this case (5.3) is always impossible, no matter what $p>1$ is. The preceding method shows therefore that the equation $-\boldsymbol{u}^{\prime \prime}=$ $|u|^{p-1} u+h(x), x \in(0,1)$ and $u(0)=u(1)=0$, has infinitely many distinct solutions, for any $p>1$ and any $h \in L^{2}(0,1)$.

6. More general nonlinearities. In this section, we consider the following more general problem than (1.1):

$$
\left\{\begin{array}{l}
L u=g(x, u)+h(x), \quad x \in \Omega, \\
u=0 \text { on } \partial \Omega
\end{array}\right.
$$

Here again $\Omega \subset \mathbf{R}^{N}$ is a smooth and bounded domain, $L$ is now a second order selfadjoint, uniformly elliptic operator:

$$
L=-\sum_{i, j=1}^{N} \frac{\partial}{\partial x_{i}}\left(a_{i j}(x) \frac{\partial}{\partial x_{j}}\right)+b(x)
$$

with $a_{i j}=a_{j i} \in C^{1}(\bar{\Omega}), b \in C^{0}(\bar{\Omega}), b \geqslant 0$ and

$$
\sum_{i, j=1}^{N} a_{i j}(x) \xi_{i} \xi_{j} \geqslant \rho|\xi|^{2}, \quad \forall x \in \bar{\Omega}, \forall \xi \in \mathbf{R}^{N},
$$

$\rho>0$ being the ellipticity constant. $h \in L^{2}(\Omega)$ is a given function. We assume that the function $g$ satisfies the following conditions.

$$
\begin{gathered}
\left\{\begin{array}{l}
g: \bar{\Omega} \times \mathbf{R} \rightarrow \mathbf{R} \text { is a continuous function, and } g=g(x, s) \\
\text { is } C^{1} \text { and odd with respect to } s \in \mathbf{R} \text {, for all } x \in \bar{\Omega} .
\end{array}\right. \\
\left\{\begin{array}{l}
\exists \mu, s_{0}, 0<\mu<1, s_{0} \geqslant 0, \text { such that } \\
0<g(x, s) / s \leqslant \mu g_{s}^{\prime}(x, s), \forall x \in \bar{\Omega}, \forall s>s_{0} .
\end{array}\right. \\
\left\{\begin{array}{l}
\lim _{s \rightarrow+\infty} \frac{g(x, s)}{s^{p}}=q(x)>0, \quad \forall x \in \bar{\Omega}, g \in C^{0}(\bar{\Omega}), \text { the convergence } \\
\text { being uniform with respect to } x \in \bar{\Omega} ; \text { here, } 1<p<p_{N} \\
\text { where } p_{N} \text { is the largest root of }(2 N-2) p^{2}-(N+2) p-N=0 .
\end{array}\right.
\end{gathered}
$$

Let us observe that condition (6.3) readily implies (by means of an integration by parts) that

$$
\left\{\begin{array}{l}
G(x, s) \leqslant \theta g(x, s) s+C, \forall x \in \bar{\Omega}, \forall s \in \mathbf{R} \\
\text { with } \theta \in\left(0, \frac{1}{2}\right) \text { and } C>0 \text { is some constant, }
\end{array}\right.
$$

where $G(x, z)=\int_{0}^{z} g(x, s) d s$ and $\theta=\mu /(1+\mu)$. This condition (6.5) is classical and is always assumed in the works of Ambrosetti and Rabinowitz [4] and Rabinowitz [22] in order to ensure that the associated functional satisfies a condition of the type (P.S). Thus, (6.3) is a stronger hypothesis than (6.5), but, in a sense, it is a condition of the same nature. ${ }^{4}$

${ }^{4}$ Actually, this condition (6.3) could be somewhat weakened by using a method similar to the one we develop in [8] and [9]. 
The main result in this section is

TheOREM 6.1. Assume that $g$ satisfies hypotheses (6.2)-(6.4). Then, for any given $h \in L^{2}(\Omega)$, problem (6.1) possesses infinitely many distinct solutions.

The proof of this result follows the steps of that given in $\$ \S 4$ and 5 for the case $g(x, u)=|u|^{p-1} u$, and rests on Theorem 3.8. Therefore, we only carry out in detail the changes which are needed for treating the more general $g$. Let us set for $\phi, \psi \in H_{0}^{1}(\Omega)$ :

$$
a(\phi, \psi)=\sum_{i, j=1}^{N} \int_{\Omega} a_{i j}(x) \frac{\partial \phi}{\partial x_{i}} \frac{\partial \psi}{\partial x_{j}} .
$$

The norm in $H_{0}^{1}(\Omega)$ will be taken here to be

$$
\|\phi\|=\sqrt{a(\phi, \phi)}, \quad S=\left\{u \in H_{0}^{1}(\Omega) ;\|u\|=1\right\} .
$$

Define for $u \in H_{0}^{1}(\Omega)$,

$$
\begin{aligned}
I(u) & =\frac{1}{2}\|u\|^{2}-\int_{\Omega} G(x, u)-\int_{\Omega} h u, \\
I^{*}(u) & =\frac{1}{2}\|u\|^{2}-\int_{\Omega} G(x, u) .
\end{aligned}
$$

As before, we define for $u \in S$,

$$
J(u)=\max _{\lambda>0} I(\lambda u), \quad J^{*}(u)=\max _{\lambda>0} I^{*}(\lambda u) .
$$

In order to isolate clearly the role played by condition (6.4) on the growth restriction of $g$, we will replace it whenever possible in the following statements by the more general (and "usual" when $N \geqslant 3$ ) assumption.

$$
\left\{\begin{array}{l}
\text { There exists } p \text { such that } \lim _{s \rightarrow+\infty} \frac{g(x, s)}{s^{p}}=0 \text { with } 1<p<\frac{N+2}{N-2} \\
\text { if } N \geqslant 3 \text { and } 1<p \text { if } N=2 \text {, the convergence being uniform } \\
\text { with respect to } x \in \bar{\Omega} .
\end{array}\right.
$$

As is well known, (6.6) can be weakened when $N=1$ or 2 . For $N=2$, one just requires $\lim _{s \rightarrow+\infty} g(x, s) e^{-s^{2}}=0$ and, actually, when $N=1$ no growth hypothesis at all is needed.

We require the following modification of Propositions 4.1 and 4.2.

Proposition 6.2. Assume that $g$ satisfies hypotheses (6.2), (6.3) and (6.6). Then, for any $h \in L^{2}(\Omega)$, there exists $A>0$ such that $J, J^{*} \in C^{0}(S, \mathbf{R}), J, J^{*}>0$ and $J \in C^{1}\left(\tilde{J}_{A}, \mathbf{R}\right), J^{*} \in C^{1}\left(\tilde{J}_{A}^{*}, \mathbf{R}\right) . J$ and $J^{*}$ satisfy condition (P.S) $)_{A}$. Furthermore, $J^{*}$ is even and verifies the condition in Theorem 2.9 .

Proposition 6.3. Under the same hypotheses as in Proposition 6.2 for any $u \in S$ with $J(u) \geqslant A$, there exists a unique $\lambda(u)>0$ such that $J(u)=I(\lambda(u) u)$. Furthermore, the critical values of $I$ in $[A,+\infty)$ coincide with the critical values of $J$ in $[A,+\infty)$. 
Before proving these propositions, let us recall that at a point $\lambda$ such that $J(u)=I(\lambda u)$, one has

$$
\begin{gathered}
J(u)=\frac{\lambda^{2}}{2}-\int_{\Omega} G(x, \lambda u)-\lambda \int_{\Omega} h u, \\
\frac{d}{d \lambda} I(\lambda u)=\lambda-\int_{\Omega} g(x, \lambda u) u-\int_{\Omega} h u=0 .
\end{gathered}
$$

The proofs of Propositions 6.2 and 6.3 rest on the two following lemmas.

LEMMA 6.4. Under the assumptions of Proposition 6.2 let $h \in L^{2}(\Omega)$ be given. For any sequence $\left(u_{n}\right) \subset S,\left(\lambda_{n}\right) \subset \mathbf{R}^{+}$such that $J\left(u_{n}\right)=I\left(\lambda_{n} u_{n}\right)$, the following are equivalent:

(i) $J\left(u_{n}\right) \rightarrow+\infty$,

(ii) $\lambda_{n} \rightarrow+\infty$,

(iii) $u_{n} \rightarrow 0$ in $H_{0}^{1}(\Omega)$.

Proof of Lemma 6.4. Since $J\left(u_{n}\right)=I\left(\lambda_{n} u_{n}\right)$, it is clear that (i) $\Rightarrow$ (ii). Suppose that $\lambda_{n} \rightarrow+\infty$. From (6.8), we derive

$$
\lim _{n \rightarrow+\infty} \frac{1}{\lambda_{n}} \int_{\Omega} g\left(x, \lambda_{n} u_{n}\right) u_{n}=1 .
$$

It is easily checked that (6.3) implies that

$$
g(x, s) \geqslant C s^{1 / \mu}-C, \quad \forall s \geqslant 0
$$

(in the sequel $C$ denotes various positive constants). Hence,

$$
C \lambda_{n}^{(1 / \mu)-1} \int_{\Omega}\left|u_{n}\right|^{(1 / \mu)+1}-C \leqslant 1 .
$$

It follows that $u_{n} \rightarrow 0$ strongly in $L^{(1 / \mu)+1}(\Omega)$, and therefore $u_{n} \rightarrow 0$ weakly in $H_{0}^{1}(\Omega)$ (and $u_{n} \rightarrow 0$ in $L^{q}(\Omega), \forall q, 1 \leqslant q<2^{*}$ ). Thus (ii) $\Rightarrow$ (iii). Conversely, suppose $u_{n} \rightarrow 0$ weakly in $H_{0}^{1}(\Omega)$ and thus $u_{n} \rightarrow 0$ strongly in $L^{p+1}(\Omega)$ ( $p$ is defined in (6.6)). From (6.6) it follows that $G(x, s) \leqslant|s|^{p+1}+C$ and therefore

$$
J(u) \geqslant \max _{\lambda>0}\left\{\frac{\lambda^{2}}{2}-\lambda^{p+1}\|u\|_{L^{p+1}}^{p+1}-C\|u\|_{L^{p+1}}\right\}-C .
$$

Now, as $u_{n} \rightarrow 0$ in $L^{p+1}(\Omega)$, it is easily derived from (6.10) that $J\left(u_{n}\right) \rightarrow+\infty$. Hence, (iii) $\Rightarrow$ (i) and the proof of Lemma 6.4 is complete.

LEMma 6.5. There exist positive constants $\bar{\lambda}>0, \alpha, \beta>0$ and $A>0$ such that the following holds. For any $v \in L^{\infty}(\Omega) \cap S$ with $J(v) \geqslant A,(d I(\lambda v) / d \lambda)_{\mid \lambda=\lambda_{0}} \leqslant \beta$ and $\lambda_{0} \geqslant \bar{\lambda}$, then one has $d^{2} I(\lambda v) / d \lambda^{2} \leqslant-\alpha$ for all $\lambda \geqslant \lambda_{0}$.

REMARK 6.6. In the above lemma, some more regularity ( $L^{\infty}$ in addition to $\left.H_{0}^{1}(\Omega)\right)$ is required from $v$. The reason is that otherwise the function $\lambda \rightarrow I(\lambda v)$ need not be a function of class $C^{2}$. It should be noted indeed that we have not imposed here any restrictions from above on the growth of $g_{s}^{\prime}(x, s)$, and thus, $I$ is not necessarily a $C^{2}$ functional on $H_{0}^{1}(\Omega)$. When $v \in L^{\infty}(\Omega) \cap S$, however, then clearly $I(\lambda v)$ is a $C^{2}$ function of $\lambda$. 
Proof of Lemma 6.5. For $v \in L^{\infty}(\Omega) \cap S$, one has

$$
\begin{aligned}
& \frac{d}{d \lambda} I(\lambda v)=\lambda-\int_{\Omega} g(x, \lambda v) v-\int_{\Omega} h v, \\
& \frac{d^{2}}{d \lambda^{2}} I(\lambda v)=1-\int_{\Omega} g_{s}^{\prime}(x, \lambda v) v^{2} .
\end{aligned}
$$

Using (6.3) we derive

$$
\frac{d^{2}}{d \lambda^{2}} I(\lambda v) \leqslant 1-\frac{1}{\mu}+\frac{1}{\mu}\left[1-\frac{1}{\lambda} \int_{\Omega} g(x, \lambda v) v\right]+C \int_{\Omega} v^{2} .
$$

From Lemma 6.4 we know that if $A$ is sufficiently large, $v \in J_{A}$ implies

$$
C \int_{\Omega} v^{2}+\frac{1}{\lambda}\left|\int_{\Omega} h v\right| \leqslant \frac{1}{2} \alpha
$$

where $C$ is the (fixed) positive constant appearing in (6.12) and where we set $\alpha=-\frac{1}{2}(1-1 / \mu)>0$. Hence, we derive from (6.11) and (6.12) that for any $v \in J_{A}, v \in L^{\infty}(\Omega)$ and any $\lambda \geqslant \bar{\lambda}$ the following inequality is satisfied:

$$
d^{2} I(\lambda v) / d \lambda^{2} \leqslant-\frac{3}{2} \alpha+\mu^{-1} \lambda^{-1}[d I(\lambda v) / d \lambda]
$$

Now set $\beta=\frac{1}{2} \bar{\lambda} \mu \alpha$. It follows from (6.13) that, if for some $\lambda_{0} \geqslant \bar{\lambda},(d I(\lambda v) / d \lambda)_{\mid \lambda=\lambda_{0}}$ $\leqslant \beta$, then $d I(\lambda v) / d \lambda$ decreases for $\lambda \geqslant \lambda_{0}$ and therefore $d^{2} I(\lambda v) / d \lambda^{2} \leqslant-\alpha$ for all $\lambda \geqslant \lambda_{0}$.

Proofs of Propositions 6.2 AND 6.3. The proofs of the remaining parts in these propositions being the same as for Propositions 4.1 and 4.2, we will only show here that there exists $A>0$ such that for all $u \in S$ with $J(u) \geqslant A$ there exists a unique $\lambda=\lambda(u)$ such that $J(u)=I(\lambda u)$. In the case $u \in L^{\infty}(\Omega) \cap S$, this can easily be deduced from Lemmas 6.4 and 6.5. To conclude that the uniqueness of $\lambda(u)$ is also valid for any $u \in S$ we will now use Lemma 6.5 together with a density argument.

First, we observe that in Lemma $6.5, \bar{\lambda}$ and $A$ can be chosen so that for any $u \in J_{A}$ and $\lambda$ with $J(u)=I(\lambda u)$ one has $\lambda \geqslant \bar{\lambda}$. This is an easy consequence of the equivalence of (i) and (ii) in Lemma 6.4 by choosing $A$ large enough. Suppose that for some $u \in S$ with $J(u) \geqslant A$ there exist $\bar{\lambda} \leqslant \lambda_{1}<\lambda_{2}$ such that $J(u)=I\left(\lambda_{1} u\right)=$ $I\left(\lambda_{2} u\right)$. Taking a sequence $\left(v_{n}\right) \subset L^{\infty}(\Omega) \cap S$ such that $v_{n} \rightarrow u$ in $H_{0}^{1}(\Omega)$ (by density and radial projection onto $S$ ), it is easily seen by using (6.6) that the sequence of functions $\lambda \mapsto I\left(\lambda v_{n}\right)$ converges uniformly in the $C^{1}$ sense on $\lambda \in\left[\lambda_{1}, \lambda_{2}\right]$ to the function $\lambda \mapsto I(\lambda u)$. Hence, $\left(d I\left(\lambda v_{n}\right) / d \lambda\right)_{\mid \lambda=\lambda_{1}} \rightarrow 0$ as $n \rightarrow \infty$. By Lemma 6.5 it follows that for $n$ large enough, say $n \geqslant n_{0}$, one always has

$$
\frac{d^{2}}{d \lambda^{2}} I\left(\lambda v_{n}\right) \leqslant-\alpha, \quad \lambda \geqslant \lambda_{1}, n \geqslant n_{0} \text {. }
$$

Therefore,

$$
I\left(\lambda_{2} v_{n}\right)-I\left(\lambda_{1} v_{n}\right) \leqslant\left[\frac{d}{d \lambda} I\left(\lambda v_{n}\right)_{\mid \lambda=\lambda_{1}}\right]\left(\lambda_{2}-\lambda_{1}\right)-\frac{\alpha}{2}\left(\lambda_{2}-\lambda_{1}\right)^{2} .
$$

Taking the limits as $n \rightarrow+\infty$ in the above inequality yields

$$
0=I\left(\lambda_{2} u\right)-I\left(\lambda_{1} u\right) \leqslant-(\alpha / 2)\left(\lambda_{2}-\lambda_{1}\right)^{2}
$$


which is absurd. This shows that $\lambda=\lambda(u)$ is uniquely determined when $u \in J_{A}$ and the proof is thereby complete.

Next, we require more precise estimates of $\lambda(u)$ and $J(u)$ as " $J(u) \rightarrow+\infty$ ".

Lemma 6.7. Assume $g$ satisfies (6.2)-(6.4), and let $h \in L^{2}(\Omega)$ be given. Then, there exist $A_{1} \geqslant A$ and constants $0<\omega_{1} \leqslant \omega_{2}$ such that for any $u \in S$ with $J(u) \geqslant A_{1}$, one has

$$
\omega_{1} \leqslant \lambda(u)\left\{\int_{\Omega}|u|^{p+1}\right\}^{1 /(p-1)} \leqslant \omega_{2}
$$

and

$$
\omega_{1} \leqslant J(u)\left\{\int_{\Omega}|u|^{p+1}\right\}^{2 /(p-1)} \leqslant \omega_{2},
$$

where $p$ is the exponent defined in (6.4). The same inequalities hold with $J$ replaced by $J^{*}$ and $\lambda$ replaced by $\lambda^{*}$ such that $J^{*}(u)=I^{*}\left(\lambda^{*} u\right)$.

Proof. By condition (6.4) there exist constants $C>0$ and $0<\zeta_{1} \leqslant \zeta_{2}$ such that

$$
\zeta_{1}|s|^{p+1}-C \leqslant g(x, s) s \leqslant \zeta_{2}|s|^{p+1}+C, \quad \forall x \in \bar{\Omega}, \forall s \in \mathbf{R} .
$$

There exists $A_{1} \geqslant A$ such that

$$
\frac{1}{2} \leqslant \frac{1}{\lambda} \int_{\Omega} g(x, \lambda u) u \leqslant \frac{3}{2}, \quad \forall u \in \tilde{J}_{A_{1}}, \lambda=\lambda(u) .
$$

This is easily derived from (6.8) by using a contradiction argument. Thus, using (6.14) and (6.15) we derive

$$
\begin{aligned}
& \zeta_{1} \lambda^{p-1} \int_{\Omega}|u|^{p+1} \leqslant 3 / 2+C / \lambda, \\
& \zeta_{2} \lambda^{p-1} \int_{\Omega}|u|^{p+1} \geqslant 1 / 2-C / \lambda
\end{aligned}
$$

for all $u \in \tilde{J}_{A_{1}}$ and $\lambda=\lambda(u)$. This yields the first inequality in Lemma 6.7. For the second inequality, we observe that $\lim _{s \rightarrow \pm \infty} G(x, s)=+\infty$ uniformly with respect to $x \in \bar{\Omega}$. Hence, for $u \in \tilde{J}_{A}$,

$$
J(u) \leqslant \lambda^{2} / 2+C-\lambda \int_{\Omega} h u
$$

with $\lambda=\lambda(u)$. Therefore, by choosing $A_{1}$ large enough so that $\lambda=\lambda(u)$ is also large enough, we derive

$$
J(u) \leqslant \frac{2}{3} \lambda^{2} \quad \forall u \in \tilde{J}_{A_{1}}, \lambda=\lambda(u) .
$$

From (6.5) it follows that

$$
J(u) \geqslant \lambda^{2}\left(\frac{1}{2}-\theta\right)+\theta \lambda^{2}\left\{1-\frac{1}{\lambda} \int g(x, \lambda u) u\right\}-C .
$$

By refining (6.15) it is seen that for any $\varepsilon>0, \varepsilon<1$, there exists $A(\varepsilon)>0$ such that

$$
1-\varepsilon \leqslant \frac{1}{\lambda} \int_{\Omega} g(x, \lambda u) u \leqslant 1+\varepsilon, \quad \forall u \in \tilde{J}_{A(\varepsilon)}, \lambda=\lambda(u)
$$


Hence, using (6.18) and $A_{1}$ sufficiently large we derive from (6.17) the existence of $\eta>0$ such that

$$
J(u) \geqslant \eta \lambda^{2}, \quad \forall u \in \tilde{J}_{A_{1}}, \lambda=\lambda(u) .
$$

Lastly, comparing (6.16), (6.19) and the first inequality of Lemma 6.7 yields the second inequality.

Our next estimate before proving Theorem 6.1 will consist in checking that Lemma 4.4 continues to hold in the present situation.

Lemma 6.8. Assume $g$ satisfies (6.2)-(6.4), and let $h \in L^{2}(\Omega)$ be given. Then there exist $A_{2} \geqslant A_{1}$ and a constant $\gamma>0$ such that for all $u \in S$ with either $J(u) \geqslant A_{2}$ or $J^{*}(u) \geqslant A_{2}$ one has

$$
\begin{aligned}
& \left|J(u)-J^{*}(u)\right| \leqslant \gamma[J(u)]^{1 /(p+1)}, \\
& \left|J(u)-J^{*}(u)\right| \leqslant \gamma\left[J^{*}(u)\right]^{1 /(p+1)}
\end{aligned}
$$

where $p$ is the exponent defined in (6.4).

Proof. Firstly, we choose $A_{2} \geqslant A_{1}$ by requiring that $J(u) \geqslant A_{2} \Rightarrow J^{*}(u) \geqslant A_{1}$ and $J^{*}(u) \geqslant A_{2} \Rightarrow J(u) \geqslant A_{1}$. (This is possible by Lemma 6.4.) Next, in exactly the same way as in the proof of Lemma 4.4 we have

$$
\left|J(u)-J^{*}(u)\right| \leqslant \max \left\{\lambda(u), \lambda^{*}(u)\right\}\|u\|_{L^{p+1}(\Omega)}\|h\|_{L^{q}(\Omega)},
$$

where $J(u)=I(\lambda(u) u)$ and $J^{*}(u)=I^{*}\left(\lambda^{*}(u) u\right)$. Using Lemma 6.7, we then deduce

$$
\left|J(u)-J^{*}(u)\right| \leqslant C\|u\|_{L^{p}(1)}^{-2 /(p-1)}, \quad \forall u \in \tilde{J}_{A_{2}} \cup \tilde{J}_{A_{2}}^{*} \text {. }
$$

Hence, we derive the inequalities in Lemma 6.8 by applying the second inequality in Lemma 6.7.

Now, as in (3.2) we define the numbers $C_{k}$ by setting

$$
C_{k}=\inf _{A \in M_{k}} \max _{u \in A} J^{*}(u)
$$

where $M_{k}$ is the class defined in (3.1). The next results contain the few modifications required in Theorems 3.1 and 3.3 and Proposition 5.1. They assert that the same estimates derived in $\$ 5$ continue to hold for a more general $\mathrm{g}$.

Proposition 6.9. Assume $g$ satisfies (6.2)-(6.6), and let $C_{k}$ be defined in (6.20). Then, one has:

(i) $0 \leqslant C_{k} \leqslant C_{k+1}, \forall k \in \mathbf{N}$.

(ii) $\lim _{k \rightarrow+\infty} C_{k}=+\infty$.

(iii) For all $k \in \mathbf{N}$ such that $C_{k}>A, C_{k}$ is a critical value of $J^{*}$ and hence of $I^{*}$.

(iv) For all $a, b \in \mathbf{R}, k \in \mathbf{N}$ with $C_{k}<b<a<C_{k+1}$, J $J_{b}^{*}$ is not contractible to $a$ point in $J_{a}^{*}$.

Proposition 6.10. In addition to the hypotheses of Proposition 6.9, assume that $g$ satisfies condition (6.4). Then, there exists a constant $C>0$ (respectively, there exists $C_{\varepsilon}>0$ for any $\varepsilon>0$ ) and $k_{0} \in \mathbf{N}$ such that

$$
\begin{cases}C_{k} \geqslant C k^{\alpha_{N}} & \text { if } N \geqslant 3, \forall k \geqslant k_{0}, \\ C_{k} \geqslant C_{\varepsilon} k^{\alpha_{2}-\varepsilon} & \text { if } N=2, \forall k \geqslant k_{0},\end{cases}
$$


where

$$
\alpha_{N}=\frac{2 N-(N-2)(p+1)}{N(p-1)},
$$

$\forall N \geqslant 2$, and $k_{0} \in \mathbf{N}$.

Proposition 6.9 repeats results from $\S 3$. Note that since $J^{*}$ is $C^{1}$ only on the set $\tilde{J}_{A}^{*}$ and only satisfies (P.S $)_{A}$, we need to know that $C_{k}>A$ in order to ensure that $C_{k}$ is a critical value. Also observe that (ii) is derived from Theorem 3.3 (i.e. with the same proof) by using Lemma 6.4 .

Proof of Proposition 6.10. It rests on the fact that $J^{*}$ satisfies the following inequality

$$
J^{*}(u) \geqslant C\left(\int_{\Omega}|u|^{p+1}\right)^{-2 /(p-1)}-C^{\prime}, \quad \forall u \in S .
$$

Indeed, by Lemma 6.4 we know that on the set $\left\{u \in S ; 0 \leqslant J^{*}(u) \leqslant A\right\}, \int_{\Omega}|u|^{p+1}$ is bounded away from 0 so that (6.22) holds on this set provided $C^{\prime}$ is large enough. On the set $\left\{u \in S ; J^{*}(u) \geqslant A\right\}$ on the other hand, (6.19) is just derived from Lemma 6.7. Hence, by repeating the same proof as for Proposition 5.1, we derive

$$
\begin{aligned}
& C_{k} \geqslant C k^{\alpha_{N}}-C^{\prime}, \quad \forall k \geqslant 1, \text { if } N \geqslant 3, \\
& C_{k} \geqslant C_{\varepsilon} k^{\alpha_{2}-\varepsilon}-C^{\prime}, \quad \forall k \geqslant 1, \text { if } N=2 .
\end{aligned}
$$

Then (6.21) follows by choosing $k_{0}$ sufficiently large.

The proof of Theorem 6.1 is now complete. Indeed, we have now the estimates (Lemma 6.8 and Proposition 6.10) that enable us to repeat exactly the same argument as in $\S 5$ for Theorem 1.1 and derive Theorem 6.1 from Theorem 3.8.

REMARK 6.11. In the odd case, that is when $h \equiv 0$, the preceding argument shows the existence of infinitely many distinct solutions of the equation

$$
\left\{\begin{array}{l}
L u=g(x, u), \quad x \in \Omega, \\
u=0 \text { on } \partial \Omega,
\end{array}\right.
$$

under assumptions (6.2), (6.3) and (6.6) only. (Condition (6.4) is not needed here.) Indeed, this is precisely the content of Propositions 6.3 for $J^{*}$ (ii) and (iii). This result is slightly weaker than the one established by Ambrosetti and Rabinowitz [22] for (6.20), who only assume (6.5) rather than (6.3) and only assume $g$ to be continuous. On the other hand, the preceding method provides in this case a somewhat simpler proof of this result. Here, the proof only relies on the study of critical points of $J^{*}$ by means of classical inf-max characterizations of the $C_{k}$ rather than on the "dual variational" approach used in [22].

REMARK 6.12. Specializing the preceding method to problem (6.1) in dimension $N=1$, we derive a result that seems new. Indeed, consider the problem

$$
\left\{\begin{array}{l}
\left(-a y^{\prime}\right)^{\prime}+b y=g(x, y)+h(x), \quad x \in(0,1), \\
y(0)=y(1)=0
\end{array}\right.
$$

where $a \in C^{1}[0,1], a>0$ on $[0,1], b \in C^{9}[0,1]$ and $b \geqslant 0$. Here, $g$ satisfies assumptions (6.2), (6.3) and (6.4), but in (6.4) $1<p<p_{N}$ is not required any longer 
and is replaced merely by $1<p<+\infty$. Then, the method of this section shows that for any $h \in L^{2}[0,1]$, problem (6.21) has infinitely many solutions. The proof in this case requires the more precise estimate for the $C_{k}$ which is indicated in Remark 5.2. The theorems (and methods) of Ehrmann [14] and Fučik and Lovicar [15] concern the O.D.E. (see the Introduction)

$$
\left\{\begin{array}{l}
-y^{\prime \prime}=f(y)+h(x), \quad x \in(0,1) \\
y(0)=y(1)=0
\end{array}\right.
$$

That is, a perturbation by $h(x)$ of an autonomous equation. However, we recall on the other hand that the condition they impose of $f$, viz. $\lim _{y \rightarrow \pm \infty}(f(y) / y)=+\infty$ is quite general in this case.

7. A perturbation and multiplicity result. In this section we study a more general perturbation problem for which we obtain a perturbation and multiplicity type result that generalizes an earlier work in this direction by A. Ambrosetti [2]. We consider the problem

$$
\left\{\begin{array}{l}
L u=g(x, u)+\varepsilon \psi(x, u), \quad x \in \Omega, \\
u=0 \text { on } \partial \Omega .
\end{array}\right.
$$

We assume that $\Omega \subset \mathbf{R}^{N}$ and $L$, a second order selfadjoint elliptic operator, are as in the preceding section. The function $g: \bar{\Omega} \times \mathbf{R} \rightarrow \mathbf{R}$ is supposed to satisfy conditions (6.2), (6.3) and (6.6). (Condition (6.4) will not be used any longer in the sequel.) $\varepsilon>0$ designates a real "perturbation parameter". We assume that $\psi$ satisfies the following conditions:

$\psi: \bar{\Omega} \times \mathbf{R} \rightarrow \mathbf{R}$ is continuous and $\psi=\psi(x, s)$ is $C^{1}$ with respect to $s \in \mathbf{R}$;

$$
\lim _{s \rightarrow \pm \infty} \frac{\psi_{s}^{\prime}(x, s)}{g_{s}^{\prime}(x, s)}=0, \quad \text { uniformly with respect to } x \in \bar{\Omega} \text {. }
$$

The preceding condition asserts that in an appropriate sense, $\psi$ is "dominated" by $g$ as $|s| \rightarrow+\infty$. The main result of this section is the following.

TheOREM 7.1. Assume $g$ satisfies conditions (6.2), (6.3) and (6.6) and that $\psi$ satisfies (7.2) and (7.3). Then, for any $\nu \in N$, there exists $\varepsilon_{\nu}>0$ such that for any $\varepsilon$, $0 \leqslant \varepsilon \leqslant \varepsilon_{\nu}$, problem (7.1) admits at least $\nu$ distinct solutions.

The proof will make use of the same setting as in $\S 6$. We recall that $H=H_{0}^{1}(\Omega)$, $\|u\|^{2}=\int_{\Omega} L u \cdot u$,

$$
I^{*}(u)=\frac{1}{2}\|u\|^{2}-\int_{\Omega} G(x, u), \quad u \in H_{0}^{1}(\Omega)
$$

with $G(x, z)=\int_{0}^{z} g(x, s) d s$ and

$$
J^{*}(u)=\max _{\lambda>0} I^{*}(\lambda u), \quad u \in S .
$$

We define $\Psi(x, z)=\int_{0}^{z} \psi(x, s) d s$,

$$
H^{e}(u)=I^{*}(u)-\varepsilon \int_{\Omega} \Psi(x, u) \quad \text { and } \quad K^{e}(u)=\max _{\lambda>0} H^{e}(\lambda u) .
$$


We require the following adaptations of Propositions 4 and 5. (Recall that $J^{*}$ and $I^{*}$ have the properties described in those propositions.)

Proposition 7.2. Assume that $g$ verifies the hypotheses of Theorem 7.1 and let $\varepsilon_{0}>0$ be given. There exists $A>0$ such that for any $\varepsilon, 0 \leqslant \varepsilon \leqslant \varepsilon_{0}, K^{\varepsilon} \in C^{0}(S, \mathbf{R})$, $K^{\varepsilon} \in C^{1}\left(\left(\widetilde{K^{\varepsilon}}\right)_{A}, \mathbf{R}\right), K^{\varepsilon}$ satisfies condition (P.S $)_{A}$. Furthermore, the critical values of $K^{e}$ in $[A,+\infty)$ coincide with the critical values of $H^{\varepsilon}$ in $[A,+\infty)$ for any $\varepsilon \in\left[0, \varepsilon_{0}\right]$.

As usual, the key for Proposition 7.2 rests on the fact that for $A$ large enough, there is a unique $\lambda=\lambda_{\varepsilon}(u)$ such that $K^{\varepsilon}(u)=H^{\varepsilon}(\lambda u) \geqslant A$ for any $\varepsilon \in\left[0, \varepsilon_{0}\right]$ and $u \in\left(\widetilde{K^{\varepsilon}}\right)_{A}$. This will be derived from the next two lemmas.

Lemma 7.3. Under the assumptions of Proposition 7.2 for any $\varepsilon \in\left[0, \varepsilon_{0}\right]$ and any sequences $\left(u_{n}\right) \subset S,\left(\lambda_{n}\right) \subset \mathbf{R}^{+}$such that $K^{\varepsilon}\left(u_{n}\right)=H^{\varepsilon}\left(\lambda_{n} u_{n}\right)$, the following are equivalent.

(i) $K^{\varepsilon}\left(u_{n}\right) \rightarrow+\infty$,

(ii) $\lambda_{n} \rightarrow+\infty$,

(iii) $u_{n} \rightarrow 0$ weakly in $H_{0}^{1}(\Omega)$.

The proof of Lemma 7.3 can be adapted from the proof of Lemma 6.4 with no difficulty by observing that from (7.3) it follows that

$$
\left\{\begin{array}{c}
\forall \alpha>0, \exists C_{\alpha}>0 \text { such that } \forall x \in \bar{\Omega}, \forall s \in \mathbf{R} ; \\
-\alpha g_{s}^{\prime}(x, s)-C_{\alpha} \leqslant \psi_{s}^{\prime}(x, s) \leqslant \alpha g_{s}^{\prime}(x, s)+C_{\alpha} ; \\
-\alpha g(x, s) s-C_{\alpha} \leqslant \psi(x, s) s \leqslant \alpha g(x, s) s+C_{\alpha} .
\end{array}\right.
$$

It just suffices to observe that by choosing $\alpha$ appropriately small, the same type of inequalities that we used for proving Lemma 6.4 continue to hold in the present situation.

LEMMA 7.4. Under the assumptions of Proposition 7.2, there exists $\bar{\lambda}$ such that for any $\varepsilon \in\left[0, \varepsilon_{0}\right], u \in S \cap L^{\infty}$ and any $\lambda \geqslant \bar{\lambda}$ with $d H^{e}(\lambda u) / d \lambda=0$, one has $d^{2} H^{\varepsilon}(\lambda u) / d \lambda^{2}<0$.

The proof of this lemma closely follows that of Lemma 6.5 and will be omitted here. (To adapt the proof of Lemma 6.5, one uses (7.4) with an adequate choice of a.)

Proof of Proposition 7.2. As in $\S 6$, the preceding lemmas show that there exists $A \geqslant 0$ such that for any $\varepsilon \in\left[0, \varepsilon_{0}\right]$ and any $u \in S$ with $K^{\varepsilon}(u)>A$, there is a unique $\lambda=\lambda_{\varepsilon}(u)$ such that $K^{\varepsilon}(u)=H^{\varepsilon}(\lambda u)$. This $\lambda_{e}(u)$ is easily seen to be a $C^{1}$ function on $\left(K^{\varepsilon}\right)_{A}$.

Then Proposition 7.2 follows in very much the same way as Proposition 4.1 was derived in $\$ 4$ and details are omitted here. In particular, condition (P.S) $)_{A}$ for $K^{\ell}$ is derived from the fact that $H^{\varepsilon}$ satisfies an analogous (P.S) $)_{A}$ condition. To see this, we observe that

$$
H^{e}(u)=\frac{1}{2}\|u\|^{2}-\int_{\Omega} G^{e}(x, u)
$$


where $G^{e}=G+\varepsilon \Psi$ and $g^{e}=g+\varepsilon \psi$. Now using (6.5) and (7.7) it is easily seen that $g^{\varepsilon}$ satisfies

$$
G^{\varepsilon}(x, s) \leqslant \theta^{\prime} g^{\varepsilon}(x, s) s+C, \quad \forall \varepsilon \in\left[0, \varepsilon_{0}\right]
$$

for all $x \in \bar{\Omega}$ and $s \in \mathbf{R}$ where $0<\theta<\theta^{\prime}<\frac{1}{2}$. Thus, condition (P.S)A for $H^{e}$ follows from the results of Ambrosetti and Rabinowitz [4].

In order to prove Theorem 7.1 by applying Theorem 3.8 or rather Theorem 3.9 we need to know that the level sets of $K^{\boldsymbol{e}}$ become close in some weak sense to those of $J^{*}$ as $\varepsilon \rightarrow 0$. This will be the aim of the next two lemmas.

LEMMA 7.5. For any $u \in S$ denote by $\lambda^{*}(u)$ and $\lambda^{e}(u)$ two real numbers such that $J^{*}(u)=I^{*}\left(\lambda^{*}(u) u\right)$ and $K^{e}(u)=H^{e}\left(\lambda^{e}(u) u\right)$. Then there exist constants $a, b>0$ such that

$$
\begin{aligned}
& {\left[\lambda^{*}(u)\right]^{2} \leqslant a J^{*}(u)+b,} \\
& {\left[\lambda^{\varepsilon}(u)\right]^{2} \leqslant a K^{e}(u)+b \quad\left(\varepsilon \in\left[0, \varepsilon_{0}\right]\right) .}
\end{aligned}
$$

Proof. In the sequel, we denote $\lambda^{e}=\lambda^{e}(u), \lambda^{*}=\lambda^{*}(u)$. One has

$$
J^{*}(u)=\frac{\left(\lambda^{*}\right)^{2}}{2}-\int_{\Omega} G\left(x, \lambda^{*} u\right)
$$

Hence, by using (6.5) we have

$$
J^{*}(u) \geqslant\left(\lambda^{*}\right)^{2}\left(\frac{1}{2}-\theta\right)+\theta \lambda^{*}\left\{\lambda^{*}-\int_{\Omega} g\left(x, \lambda^{*} u\right) u\right\}-C .
$$

Now, when $\lambda^{*}>0$ we know that

$$
\frac{d}{d \lambda} I^{*}(\lambda u)_{\mid \lambda=\lambda^{*}}=\lambda^{*}-\int_{\Omega} g\left(x, \lambda^{*} u\right) u=0 .
$$

Thus, $J^{*}(u) \geqslant\left(\lambda^{*}\right)^{2}\left(\frac{1}{2}-\theta\right)-C$ and the first inequality is proved. The second inequality in the lemma follows similarly by using (7.5).

LEMMA 7.6. For any $u \in S$ and any $\varepsilon \in\left[0, \varepsilon_{0}\right]$ the following estimates hold:

$$
\begin{aligned}
& \left|J^{*}(u)-K^{e}(u)\right|<\varepsilon\left(J^{*}(u)+a\right), \\
& \left|J^{*}(u)-K^{e}(u)\right|<\varepsilon\left(K^{e}(u)+a\right)
\end{aligned}
$$

where $a>0$ is some constant.

Proof. From (6.5) and (7.3) we know (compare (7.4)) that for any $\alpha>0$ there exists a constant $C_{\alpha}>0$ satisfying

$$
\left\{\begin{array}{l}
|\Psi(x, s)|<\alpha g(x, s) s+C_{\alpha} \\
|\Psi(x, s)|<\alpha g^{e}(x, s) s+C_{\alpha}
\end{array}\right.
$$

for any $x \in \bar{\Omega}, s \in \mathbf{R}, \varepsilon \in\left[0, \varepsilon_{0}\right]$ and where $g^{\ell}=g+\varepsilon \psi$. For a positive $\lambda^{*}$, one has $\lambda^{*}=\int_{\Omega} g\left(x, \lambda^{*} u\right) u$ and, similarly, if $\lambda^{\varepsilon}>0, \lambda^{\varepsilon}=\int_{\Omega} g^{e}\left(x, \lambda^{\varepsilon} u\right) u$. Thus, it follows from (7.6) that

$$
\int_{\Omega}\left|\Psi\left(x, \lambda^{*} u\right)\right| \leqslant \alpha\left(\lambda^{*}\right)^{2}+C_{\alpha} \text { and } \int_{\Omega}\left|\Psi\left(x, \lambda^{e} u\right)\right|<\alpha\left(\lambda^{e}\right)^{2}+C_{\alpha} .
$$


Therefore, using Lemma 7.5 yields (with another value of $C_{\alpha}$ )

$$
\left\{\begin{array}{l}
J^{*}(u)-K^{\varepsilon}(u) \leqslant \varepsilon \int_{\Omega} \Psi\left(x, \lambda^{*} u\right) \leqslant \varepsilon \alpha J^{*}(u)+\varepsilon C_{\alpha}, \\
K^{\varepsilon}(u)-J^{*}(u) \leqslant-\varepsilon \int_{\Omega} \Psi\left(x, \lambda^{\varepsilon} u\right) \leqslant \varepsilon \alpha K^{\varepsilon}(u)+\varepsilon C_{\alpha} .
\end{array}\right.
$$

From the preceding inequalities we also derive (for some constants $a, b>0$ ) by an adequate choice of $\alpha$ :

$$
\left\{\begin{array}{l}
J^{*}(u) \leqslant a K^{\varepsilon}(u)+b, \\
K^{\varepsilon}(u) \leqslant a J^{*}(u)+b,
\end{array} \quad \forall u \in S, \forall \varepsilon \in\left[0, \varepsilon_{0}\right] .\right.
$$

In showing the above inequalities we have assumed $\lambda^{*} \neq 0$ and $\lambda^{\varepsilon} \neq 0$. However (7.7) and (7.8) also hold when either $\lambda^{*}=0$ or $\lambda^{\varepsilon}=0$. Indeed from Lemma 7.3 it follows that $\lambda^{\varepsilon}$ is bounded on the set where $\lambda^{*}=0$, and vice-versa $\lambda^{*}$ is bounded on the set where $\lambda^{\varepsilon}=0$. (It just suffices to argue by contradiction and to use the fact that $\lambda^{e}\left(u_{n}\right) \rightarrow+\infty \Leftrightarrow \lambda^{*}\left(u_{n}\right) \rightarrow+\infty$.) Thus, $\int_{\Omega} \Psi\left(x, \lambda^{*} u\right)$ and $\int_{\Omega} \Psi\left(x, \lambda^{e} u\right)$ are bounded on each of these sets. This allows us to prove (7.7) and (7.8) directly in this case.

Combining inequalities (7.7) and (7.8) completes the proof of Lemma 7.6.

Proof of TheOrem 7.1. Lemma 7.6 shows that the following inclusions hold:

$$
J_{b}^{*} \subset K_{b+\varepsilon(b+a)}^{\varepsilon} \subset J_{b+3 \varepsilon(b+a)}^{*}
$$

for any $\varepsilon \in\left[0, \varepsilon_{0}\right], b>0 ; a$ is given by Lemma 7.6 and we have restricted ourselves to $0<\varepsilon_{0}<1$. Let $C_{k}$ be the critical values of $J^{*}$ defined by (6.20). By adapting the proof of Lemma 6.4 one can show that Lemma 7.3 can be strengthened in the following manner. For any sequence $\left(u_{n}\right) \subset S$ and $\varepsilon^{n} \subset\left[0, \varepsilon_{0}\right], K^{e^{n}}\left(u_{n}\right) \rightarrow+\infty$ if and only if $u_{n} \rightarrow 0$ weakly in $H$. We omit the details of this adaptation. (It uses for instance the fact that in (7.5), $\theta^{\prime}<\frac{1}{2}$ is independent of $\varepsilon$.) Therefore, we can apply Theorem 2.9 (see Remark 2.10): there exists $\mu(a) \geqslant a$, for all $a$ with the property that $K_{a}^{\varepsilon}$ is contractible to a point in $K_{\mu(a)}^{\varepsilon}$ for any $\varepsilon \in\left[0, \varepsilon_{0}\right]$. Hence, by Theorem 3.9 if the inclusions $J_{C_{k}+\rho}^{*} \subset K_{a}^{\varepsilon} \subset K_{a+\rho}^{\varepsilon} \subset J_{C_{k+1}-\rho}^{*}$ hold for some $\rho>0, a \in \mathbf{R}$, $k \in \mathbf{N}, \varepsilon \in\left[0, \varepsilon_{0}\right]$, then $K^{\varepsilon}$ has a critical value in $[a, \mu(a)]$.

Now we recall from Proposition 6.9 that $\lim _{k \rightarrow+\infty} C_{k}=+\infty$. For any integer $\nu \in \mathbf{N}$ therefore, there exists $\varepsilon_{\nu} \in\left[0, \varepsilon_{0}\right]$ and $\rho>0$ with the property that there are at least $\nu$ distinct integers $k_{1}<\cdots<k_{\nu}$ satisfying $A<C_{k_{1}}<C_{k_{2}}<\cdots<C_{k_{\nu}}$ and

$$
C_{k_{j}+1}-2 \rho>C_{k_{j}}+3 \varepsilon_{\nu}\left(C_{k_{j}}+\rho+a\right), \quad j=1, \ldots, \nu,
$$

(where $a$ is given by Lemma 7.6) and

$$
\mu\left(C_{k_{j}}+\rho+\varepsilon_{\nu}\left(C_{k_{j}}+\rho+a\right)\right)<C_{k_{j+1}}, \quad j=1, \ldots, \nu-1 .
$$

Denote $a_{j}=C_{k_{j}}+\rho+\varepsilon\left(C_{k_{j}}+\rho+a\right)$. Since $\mu$ can be chosen to be an increasing function, $\mu\left(a_{j}\right)<C_{k_{j+1}}$. From (7.9) and (7.10) we derive, for any $\varepsilon \in\left[0, \varepsilon_{\nu}\right]$,

$$
J_{C_{k_{j}+\rho}}^{*} \subset K_{a_{j}}^{\varepsilon} \subset K_{a_{j}+\rho}^{\varepsilon} \subset J_{C_{k_{j}+1}-\rho}^{*}
$$

Therefore, by Theorem 3.9, $K^{e}$ has at least one critical value in $\left[a_{j}, \mu\left(a_{j}\right)\right]$ (recall that $a_{j}>A$ and that Proposition 7.2 applies), for all $j=1, \ldots, \nu$. 
Since $\mu\left(a_{j}\right)<C_{k_{j+1}}<a_{j+1}$, the intervals $\left[a_{j}, \mu\left(a_{j}\right)\right]$ do not overlap.

Thereby, for any $\varepsilon, 0 \leqslant \varepsilon \leqslant \varepsilon$, problem (7.1) has at least $\nu$ distinct solutions and the proof of Theorem 7.1 is complete.

REMARK 7.7. The perturbation method developed in $\$ 3$ can also be applied to study other related problems. Propositions 6.9, 6.10 and 7.2 could serve as prototypes for the results needed to use a similar setting to those we have employed here. For instance, one can show the existence of infinitely many solutions for some nonlocal equations of the type

$$
\left\{\begin{array}{l}
L u=\left(\int_{\Omega} u^{2}\right)^{\alpha} u+h(x) \text { in } \Omega, \\
u=0 \text { on } \partial \Omega,
\end{array}\right.
$$

under the condition $0<\alpha<(N-2)^{-1}$ if $N \geqslant 3$ or $0<\alpha<\infty$ if $N=1,2$. This method also applies, e.g., in the case of the biharmonic operator and allows us under a suitable condition on $p$, to prove the existence of infinitely many solutions to equations of the type

$$
\left\{\begin{array}{l}
\Delta^{2} u=|u|^{p-1} u+h(x) \quad \text { in } \Omega \\
u=\partial u / \partial u=0 \text { on } \partial \Omega .
\end{array}\right.
$$

Lastly, let us indicate that one could consider somewhat more generally phrased conditions of the type (6.5) concerning equation (6.1). One can also obtain by this method results for more general perturbations in (6.1), i.e. equations of the type

$$
\left\{\begin{array}{l}
L u=g(x, u)+\phi(x, u) \text { in } \Omega \\
u=0 \text { on } \partial \Omega
\end{array}\right.
$$

under various suitable hypotheses on $g$ and $\phi$. As an example, if $g$ satisfies (6.2), (6.3) and $\lim _{s \rightarrow+\infty} g(x, s) s^{-p}=m>0$, with $1<p<(N+1) /(N-1)$ and $\phi$ is $C^{1}, \phi$ satisfies (7.3) with respect to $g$ and $\phi$ is such that $\int_{0}^{s} \phi(x, t) d t$ is bounded independently of $x \in \Omega$ and $s \in \mathbf{R}$, then (7.14) possesses infinitely many solutions.

\section{BIBLIOGRAPHY}

1. S. Agmon, Lectures on elliptic boundary value problems, Van Nostrand, Princeton, N. J., 1965.

2. A. Ambrosetti, A perturbation theorem for superlinear boundary value problems, Math. Res. Center, Univ. of Wisconsin-Madison, Tech. Sum. Report \# 1446, 1974.

3. On the existence of multiple solutions for a class of nonlinear boundary value problems, Rend. Sem. Mat. Univ. Padova 49 (1973), 195-204.

4. A. Ambrosetti and P. H. Rabinowitz, Dual variational methods in critical point theory and applications. J. Funct. Anal. 14 (1973), 349-381.

5. A. Bahri, Résolution générique d'une classe d'équations non linéaires, C. R. Acad. Sci. Paris Sér. A 291 (1980), 251-254.

6. press).

, Topological results on a certain class of functionals and applications, J. Funct. Anal. (in

7. A. Bahri and H. Berestycki, Points critiques de perturbations de fonctionnelles paires et applications, C. R. Acad. Sci. Paris Sér. A 291 (1980), 189-192.

8. __ Forced vibrations of superquadratic Hamiltonian systems (to appear); See also: Existence d'une infinité de solutions périodiques pour certains systèmes Hamiltoniens en présence d'un terme de contrainte, C. R. Acad. Sci. Paris. Sér. I 292 (1981), 315-318.

9. __ Existence of periodic solutions for some second order systems of nonlinear ordinary differential equations (to appear). 
10. C. V. Coffman, A minimum-maximum principle for a class of nonlinear integral equations, J. Analyse Math. 22 (1969), 391-419.

11. P. E. Conner and E. E. Floyd, Fixed point free involutions and equivariant maps, Bull. Amer. Math. Soc. 66 (1960), 416-441.

12. R. Courant and D. Hilbert, Methods of mathematical physics, Vol. I, Interscience, New York, 1953.

13. J. Dugundji, An extension of Tietze's theorem, Pacific J. Math. 1 (1951), 353-367.

14. H. Ehrmann, Über die Existenz der Lösungen von Randwertaufgaben bei gewöhnlicher nichtlinearen Differentialgleichungen zweiter Ordnung, Math. Ann. 134 (1957), 167-194.

15. S. Fučik and V. Lovicar, Periodic solutions of the equation $x^{\prime \prime}(t)+g(x(t))=p(t)$, Casopis Pěst. Mat. 100 (1975), 160-175.

16. J. A. Hempel, Multiple solutions for a class of nonlinear elliptic boundary value problems, Indiana Univ. Math. J. 20 (1971), 983-996.

17. M. A. Krasnosel'skii, Topological methods in the theory of nonlinear integral equations, Macmillan, New York, 1964.

18. A. Marino and G. Prodi, Metodi perturbativi nella teoria di Morse, Boll. Un. Mat. Ital. (4) 11 (1975), 1-32.

19. R. S. Palais, Ljusternik-Schnirelmann theory on Banach manifolds, Topology 5 (1966), 115-132.

20. Critical point theory and the minimax principle, Proc. Sympos. Pure Math., vol. 15, Amer. Math. Soc., Providence, R. I., 1970, pp. 185-212.

21. P. H. Rabinowitz, Variational methods for nonlinear eigenvalue problems, Indiana Univ. Math. J. 23 (1974), 729-754.

22. __ Variational methods for nonlinear eigenvalue problems, Eigenvalues of Non-linear problems, C.I.M.E., Ediz. Cremonese, Rome, 1974.

Centre National de la Récherche Scientifique et Analyse Numérique, Université Pierre et Marie Curie, 75230 Paris Cedex 05, France 